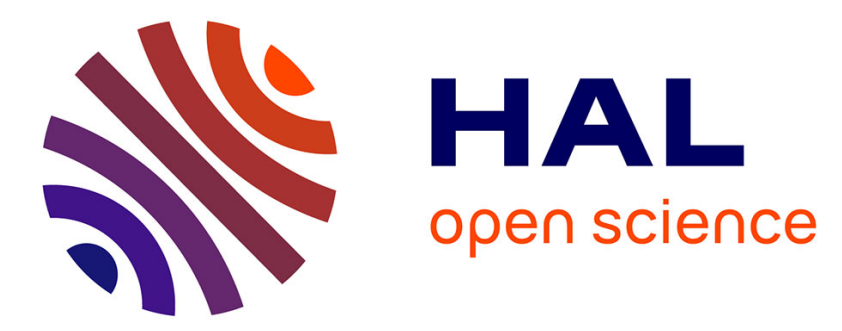

\title{
A review on hydrate composition and capability of thermodynamic modeling to predict hydrate pressure and composition
}

Saheb Maghsoodloo Babakhani, Baptiste Bouillot, Son Ho-Van, Jérome

Douzet, Jean-Michel Herri

\section{To cite this version:}

Saheb Maghsoodloo Babakhani, Baptiste Bouillot, Son Ho-Van, Jérome Douzet, Jean-Michel Herri. A review on hydrate composition and capability of thermodynamic modeling to predict hydrate pressure and composition. Fluid Phase Equilibria, 2018, 472, pp.22-38. 10.1016/j.fluid.2018.05.007 . hal01807219

\section{HAL Id: hal-01807219 https://hal.science/hal-01807219}

Submitted on 4 Jun 2018

HAL is a multi-disciplinary open access archive for the deposit and dissemination of scientific research documents, whether they are published or not. The documents may come from teaching and research institutions in France or abroad, or from public or private research centers.
L'archive ouverte pluridisciplinaire HAL, est destinée au dépôt et à la diffusion de documents scientifiques de niveau recherche, publiés ou non, émanant des établissements d'enseignement et de recherche français ou étrangers, des laboratoires publics ou privés. 
1 A review on hydrate composition and capability of thermodynamic modeling to predict hydrate pressure and composition

3 Saheb Maghsoodloo Babakhani ${ }^{*}$, Baptiste Bouillot, Son Ho-Van, Jérôme Douzet, Jean-Michel 4 Herri

5 Ecole des Mines de Saint-Etienne, SPIN, CNRS 5307, LGF, F-42023 Saint-Etienne, France

$6 \quad *$ Corresponding author: saheb.m@emse.fr

7 Keywords: gas hydrates, hydrate composition, thermodynamics, phase equilibria, modeling

9 Abstract

Gas hydrates are widely considered to be a crucial topic in oil and gas industries and attracting significant research due to potential applications such as gas storage, separation as well as water desalination. While the guest composition of hydrate phase is vital, due to the experimental difficulties in measuring hydrate composition, very little applicable information is available in the literature. Paradoxically, this is true, in spite of that; completing an experimental database on hydrate composition could have a significant impact on the processes design and modeling. Moreover, this should provide fundamental knowledge of kinetic effects as well as clarifying thermodynamic equilibrium. Hence, this paper was planned with the intent to fill in the gaps, classify and offer an overview of experimentally derived data on hydrate composition for literature. In addition, a thermodynamic model based on the van der Waals and Platteeuw approach and Kihara potential was utilized to simulate the hydrate composition along with equilibrium pressure.

Previous experimental data shows that guest distribution in hydrate phase depends noticeably on the guest composition in vapor phase. In addition, composition of larger molecules, such as propane or butane, in the hydrate phase, is notably higher than in vapor phase. Our simulation results demonstrated that the hydrate composition data from direct measurement (microscopic tools) have been well evaluated by the thermodynamic model. Nevertheless, when structural transition can occur in a system, the thermodynamic model is no longer accurate. In the case of indirect measurements, the thermodynamic model usually predicts well the hydrate composition. Nonetheless, its capability does vary with differing hydrate composition and equilibrium pressure, to the extent that in some cases, it completely fails to predict hydrate composition. This could be due to kinetic effects on the enclathration of guest molecules during the crystallization, errors in experimental techniques to measure the hydrate composition or the model parameters like Kihara potential are not properly applied. Finally, these observations show that more reliable experimental database is needed to study the evolution of guest distribution in hydrate phase and some enhancements are required for the standard thermodynamic model. 


\section{Introduction}

37 One of the main concerns in flow assurance during oil and gas production is avoiding gas hydrate formation due to the presence of water and light hydrocarbons at high pressure and low temperature conditions, especially true for deep sea drilling or artic exploration and pipelines. Therefore, the safety and economic costs of removing gas hydrates have been of great interest [18]. Plus, the enormous amount of natural gas in the form of oceanic and permafrost gas hydrates are a promising future energy resource [9-12]. Recently, gas hydrates have attracted widespread attention due to diverse applications such as gas separation [13-21], $\mathrm{CO}_{2}$ capture [22-30], water desalination [31-38], natural gas storage and transportation [39-44] and even planetary science [45-48]. For all above mentioned applications of clathrate hydrates, it is essential to increase our knowledge of thermodynamics, kinetics and the crystallization mechanisms of clathrate hydrates.

47 Numerous studies have investigated phase equilibria of clathrate hydrates [12,33,49-54].

48 Nonetheless, there has been little consideration for hydrate composition. Indeed, quantifying hydrate equilibrium composition presents several obstacles such as water occlusion, heterogeneous solid phase and measuring of solid phase concentration [12]. Hence, experimental data on hydrate composition has been rare. However, these publications do include some valuable information about fundamental hydrate phase composition, enclathration process as well as guest distribution in hydrate phase. Thus, we were able to collect invaluable data from the literature about the guest distribution in hydrate phase over a wide range of temperature, pressure and compositions. Furthermore, a thermodynamic model based on van der Waals and Platteeuw approach (vdWP) was used to detect divergences from this standard approach and previous experiments. Differences will be discussed, considering that experimental data may not be at thermodynamic equilibrium.

\section{9}

\section{Gas hydrates}

Water molecules in presence of gas molecules at high pressure and low temperature can form cavities and guest molecules like $\mathrm{CO}_{2}, \mathrm{~N}_{2}, \mathrm{CH}_{4}, \mathrm{C}_{2} \mathrm{H}_{6}$ etc. can be encased into these cavities. This ice-like structure is called gas hydrate. Based on the type of cavities and also the nature of guest molecules, there are three principal structures, sI, sII and sH. The sI usually forms in presence of small molecules (diameter between 4.2 and $6 \AA$ ) like methane and carbon dioxide. Larger molecules (diameter between 6 and $7 \AA$ ) like propane, or small molecules (diameter less than 4.2 $\AA$ ), such as nitrogen, form sII. sH is composed of a combination of small and large molecules (typically between 7 and $9 \AA$ ) $[1,2]$. Table 1 presents the differences between structures. 


\begin{tabular}{|c|c|c|c|c|c|c|c|}
\hline Hydrate structures & \multicolumn{2}{|c|}{$\mathrm{sI}$} & \multicolumn{2}{|c|}{ sII } & \multicolumn{3}{|c|}{$\mathrm{sH}$} \\
\hline Shape & \multicolumn{2}{|c|}{ 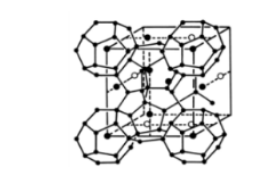 } & \multicolumn{2}{|c|}{ sns } & \multicolumn{3}{|c|}{ in } \\
\hline Cavity & Small & Large & Small & Large & Small & Medium & Large \\
\hline Description & $5^{12}$ & $5^{12} 6^{2}$ & $5^{12}$ & $5^{12} 6^{4}$ & $5^{12}$ & $4^{3} 5^{6} 6^{3}$ & $5^{12} 6^{8}$ \\
\hline Number per unit cell $\left(\mathbf{m}_{\mathrm{i}}\right)$ & 2 & 6 & 16 & 8 & 3 & 2 & 1 \\
\hline Average cavity radius $(\AA ̊)$ & 3.95 & 4.33 & 3.91 & 4.73 & 3.91 & 4.06 & 5.71 \\
\hline Coordination number ${ }^{\text {a }}$ & 20 & 24 & 20 & 28 & 20 & 20 & 36 \\
\hline
\end{tabular}

\section{3. Thermodynamic modeling}

73 At thermodynamic equilibrium, the chemical potential of each phase must be the same. In the case of gas hydrates, based on the classical van der Waals and Platteeuw model [55], liquidhydrate equilibrium is expressed by defining a metastable phase $\beta$, corresponding to the empty

76 hydrate:

$77 \Delta \mu_{W}^{L-\beta}=\Delta \mu_{W}^{H-\beta}$

78 The left hand side of equation 1 is the difference between chemical potential of water in liquid and $\beta$ phase and the right hand side is the difference between chemical potential of water in

80 hydrate and $\beta$ phase.

\section{3.1. Description of $\Delta \mu_{W}^{L-\beta}$}

82 The difference between chemical potential of water in liquid phase and $\beta$ phase can be described

83 by classical thermodynamic using Gibbs-Duhem equation. It can be rewritten as follow [14]:

$\Delta \mu_{W}^{L-\beta}=$

$85 T \frac{\left.\Delta \mu_{W}^{L-\beta}\right|_{T^{0} P^{0}}}{T^{0}}+\left(b_{P, W}^{L-\beta} T^{0}-\left.\Delta C_{p, w}^{L-\beta}\right|_{P^{0} T^{0}}\right)-T \ln \frac{T}{T^{0}}+\frac{1}{2} b_{P, W}^{L-\beta} T\left(T-T^{0}\right)+\left(\left.\Delta h_{W, m}^{L-\beta}\right|_{P^{0} T^{0}}+\right.$

$$
\left.T^{0}\left(b_{P, W}^{L-\beta} T^{0}-\left.\Delta C_{p, w}^{L-\beta}\right|_{P^{0} T^{0}}\right)-\frac{1}{2} b_{P, W}^{L-\beta} T^{0^{2}}\right)\left(1-\frac{T}{T^{0}}\right)+\left.\Delta v_{W, m}^{L-\beta}\right|_{T^{0}}\left(P-P^{0}\right)-R T \ln x_{W}^{L}
$$

87 where $T^{0}=273.15 \mathrm{~K}$ and $P^{0}=0$ are the reference temperature and pressure, respectively. $\Delta v_{W, m}^{L-\beta}$, $\Delta C_{p, w}^{L-\beta}$ and $b_{P, W}^{L-\beta}$ are thermodynamic properties of water in liquid phase and $\beta$ phase and they were calculated by Sloan and Koh [12]. $\Delta h_{W, m}^{L-\beta}$ and $\Delta \mu_{W}^{L-\beta}$ are microscopic parameters of hydrates and regrettably there are different values correspond to each author. Based on a previous 
modeling gas hydrates equilibrium [14,56]. All parameters are listed in Table 2. More details can

93 be found in the previous works of our group [14,47,57,58].

Table 2. Thermodynamic properties of hydrates

\begin{tabular}{|c|c|c|c|}
\hline Parameters & Unit & Structure I & Structure II \\
\hline$\Delta h_{W, m}^{L-\beta}$ & $\mathrm{J} / \mathrm{mol}$ & -5080 & -5247 \\
\hline$\Delta v_{W, m}^{L-\beta}$ & $10^{-6} \mathrm{~m}^{3} / \mathrm{mol}$ & 4.5959 & 4.99644 \\
\hline$\Delta C_{p, w}^{L-\beta}$ & $\mathrm{J} / \mathrm{mol} / \mathrm{K}$ & -38.12 & -38.12 \\
\hline$b_{P, W}^{L-\beta}$ & $\mathrm{J} / \mathrm{mol} / \mathrm{K}^{2}$ & 0.141 & 0.141 \\
\hline$\Delta \mu_{W}^{L-\beta}$ & $\mathrm{J} / \mathrm{mol}$ & 1287 & 1068 \\
\hline
\end{tabular}

95

$96 \quad$ 3.2. Description of $\Delta \mu_{W}^{H-\beta}$

97 From van der Waals and Platteeuw approach, statistical thermodynamics is used to express $98 \Delta \mu_{W}^{H-\beta}$ :

$99 \Delta \mu_{W}^{H-\beta}=R T \sum_{i} v_{i} \ln \left(1-\sum_{j} \theta_{j}^{i}\right)$

100 where $v_{i}$ is the number of cavities type $i$ per mole of water. $\theta_{j}^{i}$ is the occupancy factor of 101 molecule $j$ in the cavity $i$. The occupancy factor can be described by considering the analogy 102 between gas adsorption in the three dimensional hydrate structures and two-dimensional 103 Langmuir adsorption [12,59].

$104 \quad \theta_{j}^{i}=\frac{C_{j}^{i} f_{j}(T, P)}{1+\sum_{j} C_{j}^{i} f_{j}(T, P)}$

105 where $f_{j}(T, P)$ is the fugacity of guest molecule $j$ at desire temperature and pressure. The value of 106 fugacity can be calculated by assuming equilibrium with a gas phase. Therefore, a standard 107 equation of state, such as Soave-Redlich-Kwong (SRK), can be used as fugacities are similar in 108 all phases, including vapor phase. $C_{j}^{i}$ is the Langmuir constant of guest molecule $j$ in the cavity 109 type $i$. The Langmuir constant depends on the interaction potential between the trapped guest 110 molecules and the surrounding water molecules cage, and for spherical guest-cages potentials can 111 be expressed as follows:

$112 C_{j}^{i}=\frac{4 \pi}{k T} \int_{0}^{\infty} \exp \left(-\frac{w(r)}{k T}\right) r^{2} d r$

113 where $w(r)$ is the potential interaction between the guest molecule and the cavity based on the 114 distance between the gas and water molecule in the structure $(r)$. In our study, the potential 115 interaction was calculated based on the Kihara parameters as following:

$116 \quad w(r)=2 z \varepsilon\left[\frac{\sigma^{12}}{R^{11} r}\left(\Delta^{10}+\frac{a}{R} \Delta^{11}\right)-\frac{\sigma^{6}}{R^{5} r}\left(\Delta^{4}+\frac{a}{R} \Delta^{5}\right)\right]$ 
and

$118 \Delta^{N}=\frac{1}{N}\left[\left(1-\frac{r}{R}-\frac{a}{R}\right)^{-N}-\left(1+\frac{r}{R}-\frac{a}{R}\right)^{-N}\right]$

119 Parameters $\varepsilon, \sigma$ and $a$ are so-called Kihara parameters and they correspond to minimum energy, 120 collision diameter and hard-core radius, respectively. The Kihara parameters for several gases 121 were obtained in the previous studies by our group [47,57], and Sloan [60]. They are listed in 122 Table 3.

Table 3. Kihara parameters for several gases. $\varepsilon / \mathrm{k}$, $\sigma$ for $\mathrm{N}_{2}, \mathrm{CO}_{2}, \mathrm{CH}_{4}, \mathrm{C}_{2} \mathrm{H}_{6}, \mathrm{C}_{3} \mathrm{H}_{8}$ and i- $\mathrm{C}_{4} \mathrm{H}_{10}$ determined from our earlier studies $[47,57,61]$ and $\mathrm{n}-\mathrm{C}_{4} \mathrm{H}_{10}$ form Sloan [60]. Hard-core radius for all gases from Sloan [60]

\begin{tabular}{|l|c|c|c|}
\hline \multicolumn{1}{|c|}{$\mathrm{Gas}$} & $\varepsilon / \mathrm{k}(\mathrm{K})$ & $\sigma(\AA)$ & $\mathrm{a}(\AA)$ \\
\hline $\mathrm{N}_{2}$ & 133.13 & 3.099 & 0.3526 \\
\hline $\mathrm{CO}_{2}$ & 178.21 & 2.873 & 0.6805 \\
\hline $\mathrm{CH}_{4}$ & 166.36 & 3.050 & 0.3834 \\
\hline $\mathrm{C}_{2} \mathrm{H}_{6}$ & 177.46 & 3.205 & 0.5651 \\
\hline $\mathrm{C}_{3} \mathrm{H}_{8}$ & 195.00 & 3.340 & 0.6502 \\
\hline $\mathrm{i}-\mathrm{C}_{4} \mathrm{H}_{10}$ & 212.50 & 3.239 & 0.8706 \\
\hline $\mathrm{n}-\mathrm{C}_{4} \mathrm{H}_{10}$ & 209.0 & 2.9125 & 0.9379 \\
\hline
\end{tabular}

127 First, for a gas mixture at a desired temperature, the equilibrium pressure which satisfies equation 128 (1) is calculated. Subsequently, the occupancy factor is determined based on equation (4). Then, 129 the hydrate composition is calculated as following:

$130 x_{j}^{H}=\frac{\sum_{i} v_{i} \theta_{j}^{i}}{\sum_{i} \sum_{j} v_{i} \theta_{j}^{i}}$

131 The procedure for calculating hydrate equilibrium pressure and composition of mixed gas 132 hydrates is shown in Figure 1. 


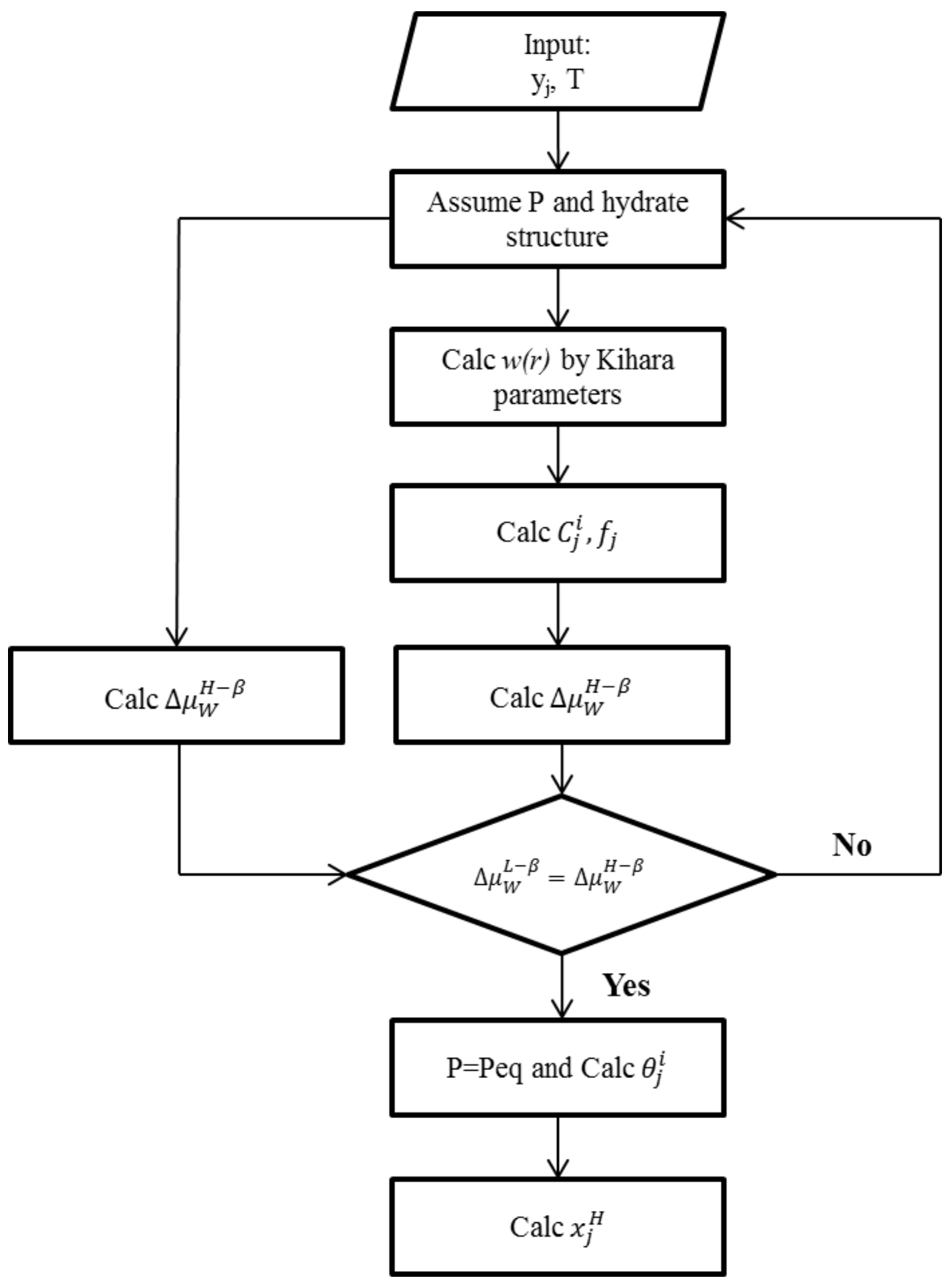

Figure 1. Procedure for calculating gas hydrate equilibrium pressure and composition

135 The average deviations for pressure and hydrate composition have been calculated based on 136 equations 9 and 10 , respectively.

$137 \quad A A D p \%=\frac{100}{N} \sum_{i}^{N}\left(\left|\frac{P_{i}^{e x p}-P_{i}^{\text {pre }}}{P_{i}^{\text {exp }}}\right|\right)$

$138 \quad A A D c=\frac{1}{N} \sum_{i}^{N}\left(\left|x_{i}^{\text {exp }}-x_{i}^{\text {pre }}\right|\right)$

139 where $i$ is equilibrium point, $N$ total number of equilibrium points, $P$ pressure, $x$ hydrate 140 composition, exp experimental data and pre prediction results. $A A D p$ and $A A D c$ are average 141 absolute deviation for pressure and composition, respectively. 
All the thermodynamic modelling section has been implemented in our in-house software, GasHyDyn. This software has shown a very good capability of liquid-hydrate equilibrium predictions [14,57], and will be used to discuss the experimental results for both pressure and hydrate composition. A photo from GasHyDyn environment with complementary information summarizing the procedure of submitting a calculation has been added to the Supporting Information document (Appendix A).

Nota Bene: the hydrate structure for modeling was chosen based on the statement of each research. Unfortunately, there were some cases that the authors did not provide the structure. Therefore, we simulated their experimental data for both structures I and II. Consequently, the structure which agreed better with the simulation results was chosen.

\section{Hydrate composition in literature and model comparison}

Despite countless works on equilibrium pressure and temperature of mixed gas hydrates, to the best of our knowledge, there are still few studies on the hydrate composition which depends on the pressure, temperature and gas phase composition. Thanks to gas chromatography, the composition of the gas phase can be easily measured, but solid phase analysis is still challenging, often leading to experimental errors. In addition, some researchers studied the hydrate composition of gas mixtures by different methods making them exceedingly difficult to compare. Hence, in the present work, studies providing hydrate composition in open literature were collected and presented. Moreover, the capability of a thermodynamic model to predict hydrate pressure and composition was evaluated. This should be noted that there were some studies on the hydrate composition of gas mixtures that do not furnish exact values of hydrate composition. Sometimes, only figures were shown and quantitative data were not available. In this section, the collected data from open literature is categorized based on their methods of hydrate composition measurements.

\subsection{Dissociation of whole hydrate phase}

One of the first systematic reports on the mixed hydrate composition was carried out by Jhaveri and Robinson [62]. They studied the gas hydrate equilibrium curves of methane-nitrogen mixture as well as the guest composition in gas and hydrate phases. They introduced gas mixture and water inside a batch reactor at a pressure $25 \%$ more than the equilibrium pressure at a desired temperature. After completion of hydrate formation, the gas phase was analyzed chromatographically. The gas was then removed from the reactor and the hydrate crystals dissociated. The decomposed gas was analyzed to obtain the hydrate composition. They measured hydrate composition at three temperatures 273.2, 277.4 and $279.8 \mathrm{~K}$ for various ranges of pressure and gas compositions. Their results are illustrated in Figure 2. Clearly, at a constant temperature, by increasing the molar composition of nitrogen in the gas phase, the equilibrium pressure increased. Since the hydrate equilibrium pressure of nitrogen at a desired temperature is 
178 higher than of methane. Moreover, the simulation results of equilibrium pressure were in a good accordance with the experimental data.

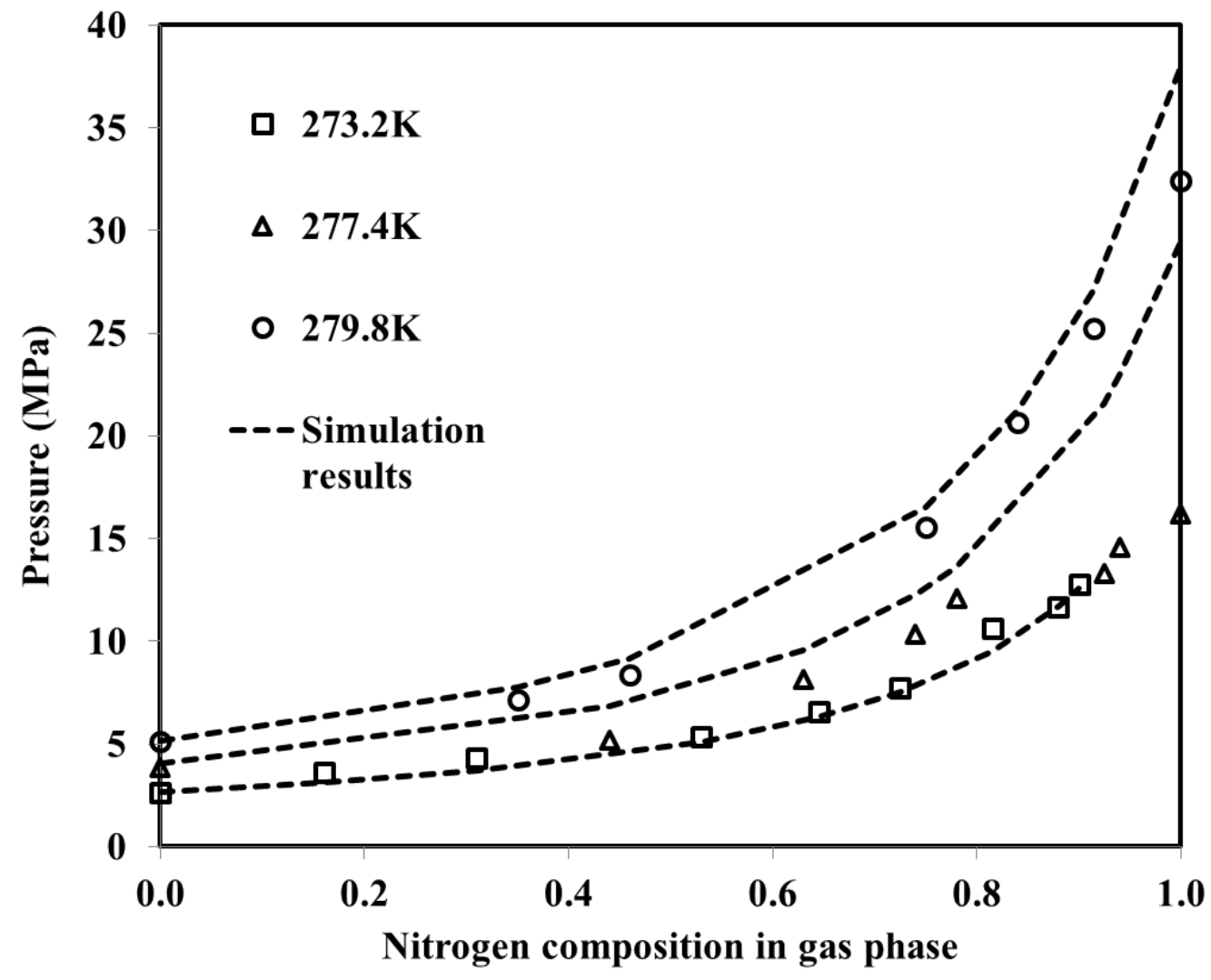

Figure 2. P-x diagram of nitrogen-methane mixed hydrate (sI) [62]

Table 4. The experimental results of Jhaveri and Robinson [62] for methane-nitrogen mixture hydrates and simulation results. Uncertainties were not provided.

\begin{tabular}{|c|c|c|c|c|c|c|c|c|c|}
\hline \multirow{2}{*}{ Texp (K) } & \multirow{2}{*}{$\begin{array}{c}\text { Pexp } \\
(\mathrm{MPa})\end{array}$} & \multirow{2}{*}{$\begin{array}{c}\text { Ppre } \\
(\mathrm{MPa})\end{array}$} & \multirow{2}{*}{$\mathrm{S}$} & \multicolumn{2}{|c|}{\begin{tabular}{c} 
Gas composition (exp) \\
\cline { 5 - 10 }
\end{tabular}} & & \multicolumn{2}{|c|}{$\begin{array}{c}\text { Hydrate composition } \\
(\mathrm{exp})\end{array}$} & \multicolumn{2}{|c|}{$\begin{array}{c}\text { Hydrate composition } \\
\text { (pre) }\end{array}$} \\
\hline 273.2 & 2.640 & 2.660 & $\mathrm{I}$ & 0.000 & 1.000 & 0.000 & 1.000 & 0.000 & 1.000 \\
\hline 273.2 & 3.620 & 3.116 & $\mathrm{I}$ & 0.160 & 0.840 & 0.065 & 0.935 & 0.034 & 0.966 \\
\hline 273.2 & 4.310 & 3.708 & $\mathrm{I}$ & 0.310 & 0.690 & 0.098 & 0.902 & 0.078 & 0.922 \\
\hline 273.2 & 5.350 & 5.092 & $\mathrm{I}$ & 0.530 & 0.470 & 0.200 & 0.800 & 0.176 & 0.824 \\
\hline 273.2 & 6.550 & 6.301 & $\mathrm{I}$ & 0.645 & 0.355 & 0.350 & 0.650 & 0.259 & 0.741 \\
\hline 273.2 & 7.750 & 7.517 & $\mathrm{I}$ & 0.725 & 0.275 & 0.425 & 0.575 & 0.339 & 0.661 \\
\hline 273.2 & 10.640 & 9.525 & $\mathrm{I}$ & 0.815 & 0.185 & 0.620 & 0.380 & 0.466 & 0.534 \\
\hline 273.2 & 11.650 & 11.716 & $\mathrm{I}$ & 0.880 & 0.120 & 0.710 & 0.290 & 0.597 & 0.403 \\
\hline 273.2 & 12.770 & 12.577 & $\mathrm{I}$ & 0.900 & 0.100 & 0.765 & 0.235 & 0.646 & 0.354 \\
\hline 277.4 & 3.860 & 4.028 & $\mathrm{I}$ & 0.000 & 1.000 & 0.000 & 1.000 & 0.000 & 1.000 \\
\hline
\end{tabular}




\begin{tabular}{|l|c|c|c|c|c|c|c|c|c|}
\hline 277.4 & 5.200 & 6.839 & $\mathrm{I}$ & 0.440 & 0.560 & 0.180 & 0.820 & 0.138 & 0.862 \\
\hline 277.4 & 8.110 & 9.575 & $\mathrm{I}$ & 0.630 & 0.370 & 0.310 & 0.690 & 0.262 & 0.738 \\
\hline 277.4 & 10.340 & 12.286 & $\mathrm{I}$ & 0.740 & 0.260 & 0.470 & 0.530 & 0.378 & 0.622 \\
\hline 277.4 & 12.060 & 13.628 & $\mathrm{I}$ & 0.780 & 0.220 & 0.560 & 0.440 & 0.433 & 0.567 \\
\hline 277.4 & 13.320 & 21.582 & $\mathrm{I}$ & 0.925 & 0.075 & 0.810 & 0.190 & 0.734 & 0.266 \\
\hline 277.4 & 14.590 & 22.950 & $\mathrm{I}$ & 0.940 & 0.060 & 0.860 & 0.140 & 0.779 & 0.221 \\
\hline 277.4 & 16.210 & 29.486 & $\mathrm{I}$ & 1.000 & 0.000 & 1.000 & 0.000 & 1.000 & 0.000 \\
\hline 279.8 & 5.140 & 5.161 & $\mathrm{I}$ & 0.000 & 1.000 & 0.000 & 1.000 & 0.000 & 1.000 \\
\hline 279.8 & 7.140 & 7.751 & $\mathrm{I}$ & 0.350 & 0.650 & 0.091 & 0.909 & 0.102 & 0.898 \\
\hline 279.8 & 8.370 & 9.170 & $\mathrm{I}$ & 0.460 & 0.540 & 0.224 & 0.776 & 0.155 & 0.845 \\
\hline 279.8 & 15.550 & 16.516 & $\mathrm{I}$ & 0.750 & 0.250 & 0.550 & 0.450 & 0.404 & 0.596 \\
\hline 279.8 & 20.670 & 21.253 & $\mathrm{I}$ & 0.840 & 0.160 & 0.680 & 0.320 & 0.549 & 0.451 \\
\hline 279.8 & 25.230 & 27.054 & $\mathrm{I}$ & 0.914 & 0.086 & 0.802 & 0.198 & 0.715 & 0.285 \\
\hline 279.8 & 32.420 & 37.997 & $\mathrm{I}$ & 1.000 & 0.000 & 1.000 & 0.000 & 1.000 & 0.000 \\
\hline \multicolumn{7}{|c|}{ AADp } & $16.32 \%$ & \multicolumn{7}{|c|}{ AADc } & & & \\
\hline
\end{tabular}

185

186

187

188

189

190

191

192

193

194

195

196

197

198

199

200

201

202

203

204

205

The results of thermodynamic simulation for hydrate pressure and composition are detailed in Table 4. At $273.2 \mathrm{~K}$ and $279.8 \mathrm{~K}$, the equilibrium pressures were successfully predicted. Nonetheless, the equilibrium pressures at $277.4 \mathrm{~K}$ for nitrogen compositions more than $90 \%$ mole fraction (in gas phase) were poorly simulated (sI). As nitrogen forms sII, we suspected a phase transition in this case. Hence, the experimental data were also simulated by considering sII. The results revealed that sII simulations were also unsuccessful to predict the equilibrium pressures for the $277 \mathrm{~K}$ isotherm at high concentrations of nitrogen in the mixture of $\mathrm{CH}_{4} / \mathrm{N}_{2}$. This might be due to whether the co-existence of sI and SII at this condition (which cannot be predicted by the model as it is implemented in our software) or the experimental measurement uncertainties.

The hydrate composition predictions were slightly different. The simulation results at low and high percentages of nitrogen in the hydrate phase agreed satisfactorily with the experimental data. But when there is no significant difference between the compositions of methane and nitrogen, the simulation results deviated from experimental. Still, the average absolute deviation of hydrate composition was less than 0.07 in mole fraction.

Kawasaki et al. [63] studied the guest content in hydrate phase for a gas mixture of methane, ethane, propane and iso-butane with initial molar concentrations 0.885, 0.046, 0.054 and 0.015, respectively. They used the same procedure as Jhaveri and Robinson [62] by removing the gas and dissociating the hydrate to measure the hydrate composition at two different temperatures, 274.15 and $278.15 \mathrm{~K}$. The experimental and simulation results are presented in Table 5.

Table 5. The gas composition in different phases at 3MPa. Uncertainties were not provided.

\begin{tabular}{|c|c|c|}
\hline \multirow{2}{*}{ Gas } & $\mathrm{T}=274.15 \mathrm{~K}$ & $\mathrm{~T}=278.15 \mathrm{~K}$ \\
\cline { 2 - 3 } & Gas and hydrate phase compositions & Gas and hydrate phase compositions \\
\hline
\end{tabular}




\begin{tabular}{|l|c|c|c|c|c|c|}
\hline & Gas (exp) & $\begin{array}{c}\text { Hydrate } \\
(\exp )\end{array}$ & $\begin{array}{c}\text { Hydrate } \\
\text { (Pre) }\end{array}$ & Gas (exp) & Hydrate (exp) & Hydrate (Pre) \\
\hline Methane & 0.987 & 0.760 & 0.827 & 0.979 & 0.702 & 0.784 \\
\hline Ethane & 0.011 & 0.089 & 0.031 & 0.017 & 0.104 & 0.034 \\
\hline Propane & 0.002 & 0.117 & 0.141 & 0.004 & 0.150 & 0.181 \\
\hline iso-Butane & 0.0 & 0.034 & NA & 0.0 & 0.044 & NA \\
\hline
\end{tabular}

207 The authors remarked that all i-butane molecules concentrated in the hydrate phase, irrespective 208 of the temperature. They also considered that the concentration of heavier hydrocarbon in hydrate 209 phase at $278.15 \mathrm{~K}$ is higher at $274.15 \mathrm{~K}$. Moreover, the deviation of simulation results for the 210 hydrate composition of methane, ethane and propane were 0.074, 0.064 and 0.027, respectively. 211 The deviations between the experimental and prediction values could be due to uncertainties in 212 the experimental procedure. In fact, Kawasaki et al. removed the gas mixture from the reactor 213 after hydrate formation to measure hydrate composition. Hence, the hydrate phase could be 214 dissociated during the gas removing step due to the pressure drop, leading to the measurement 215 uncertainties.

216 Kang et al. [64] measured the hydrate composition of $\mathrm{CO}_{2}-\mathrm{N}_{2}$ mixture at three isotherms of 217 vapor-hydrate equilibrium condition. After hydrate formation, they purged the gas outside the 218 cell and dissociated the hydrate by increasing temperature. Figure 3 presents the equilibrium 219 pressure versus nitrogen composition in the gas phase at $\mathrm{H}-\mathrm{V}$ equilibrium condition for three 220 isotherms. Our simulation results are also shown by dash lines in the figure. The simulation of 221 hydrate composition is detailed in Table 6 as well as the experimental results. 


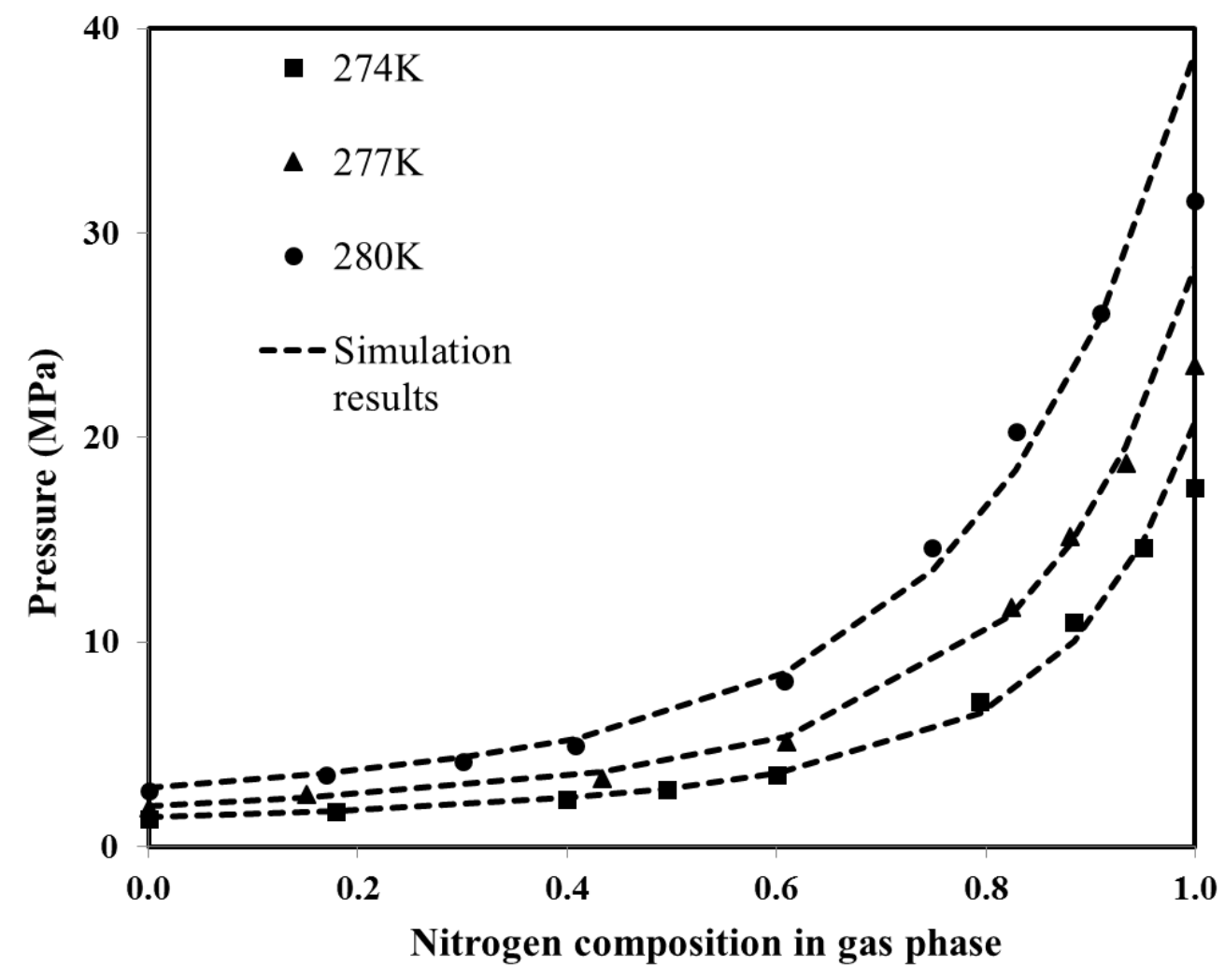

Figure 3. Experimental $\mathrm{H}-\mathrm{V}$ equilibrium data for $\mathrm{N}_{2} / \mathrm{CO}_{2}$ mixture by Kang et al. [64] and the simulation results (sI)

Table 6. Experimental results of Kang et al. [64] and simulation. Composition uncertainties were not provided.

\begin{tabular}{|c|c|c|c|c|c|c|c|c|c|}
\hline \multirow{2}{*}{$\begin{array}{c}\text { Texp }(\mathrm{K}) \\
( \pm 0.1)\end{array}$} & $\begin{array}{c}\text { Pexp } \\
(\mathrm{MPa}) \\
( \pm 0.01)\end{array}$ & \multirow{2}{*}{$\begin{array}{c}\text { Ppre } \\
(\mathrm{MPa})\end{array}$} & \multirow{2}{*}{$\mathrm{S}$} & \multicolumn{2}{|c|}{ Gas composition (exp) } & \multicolumn{2}{|c|}{$\begin{array}{c}\text { Hydrate composition } \\
(\exp )\end{array}$} & \multicolumn{2}{|c|}{$\begin{array}{c}\text { Hydrate composition } \\
\text { (pre) }\end{array}$} \\
\cline { 7 - 11 } & & & $\mathrm{CO}_{2}$ & $\mathrm{~N}_{2}$ & $\mathrm{CO}_{2}$ & $\mathrm{~N}_{2}$ & $\mathrm{CO}_{2}$ & $\mathrm{~N}_{2}$ \\
\hline 274.0 & 1.36 & 1.42 & $\mathrm{I}$ & 1.00 & 0.00 & 1.00 & 0.00 & 1.00 & 0.00 \\
\hline 274.0 & 1.73 & 1.74 & $\mathrm{I}$ & 0.82 & 0.18 & 0.99 & 0.02 & 0.98 & 0.02 \\
\hline 274.0 & 2.30 & 2.39 & $\mathrm{I}$ & 0.60 & 0.40 & 0.95 & 0.05 & 0.94 & 0.06 \\
\hline 274.0 & 2.77 & 2.84 & $\mathrm{I}$ & 0.50 & 0.50 & 0.93 & 0.07 & 0.92 & 0.08 \\
\hline 274.0 & 3.48 & 3.57 & $\mathrm{I}$ & 0.40 & 0.60 & 0.90 & 0.10 & 0.87 & 0.13 \\
\hline 274.0 & 7.07 & 6.55 & $\mathrm{I}$ & 0.21 & 0.79 & 0.58 & 0.42 & 0.71 & 0.29 \\
\hline 274.0 & 10.95 & 10.03 & $\mathrm{I}$ & 0.12 & 0.88 & 0.34 & 0.66 & 0.53 & 0.47 \\
\hline 274.0 & 14.59 & 14.91 & $\mathrm{I}$ & 0.05 & 0.95 & 0.18 & 0.82 & 0.28 & 0.72 \\
\hline 274.0 & 17.52 & 20.81 & $\mathrm{I}$ & 0.00 & 1.00 & 0.00 & 1.00 & 0.00 & 1.00 \\
\hline 277.0 & 1.91 & 1.99 & $\mathrm{I}$ & 1.00 & 0.00 & 1.00 & 0.00 & 1.00 & 0.00 \\
\hline 277.0 & 2.54 & 2.38 & $\mathrm{I}$ & 0.85 & 0.15 & 0.98 & 0.02 & 0.98 & 0.02 \\
\hline 277.0 & 3.30 & 3.65 & $\mathrm{I}$ & 0.57 & 0.43 & 0.95 & 0.05 & 0.93 & 0.07 \\
\hline
\end{tabular}




\begin{tabular}{|c|c|c|c|c|c|c|c|c|c|}
\hline 277.0 & 5.12 & 5.40 & I & 0.39 & 0.61 & 0.89 & 0.11 & 0.85 & 0.15 \\
\hline 277.0 & 11.71 & 11.30 & I & 0.18 & 0.82 & 0.54 & 0.46 & 0.62 & 0.38 \\
\hline 277.0 & 15.15 & 14.81 & I & 0.12 & 0.88 & 0.35 & 0.65 & 0.49 & 0.51 \\
\hline 277.0 & 18.74 & 19.56 & I & 0.07 & 0.93 & 0.19 & 0.81 & 0.31 & 0.69 \\
\hline 277.0 & 23.50 & 28.37 & I & 0.00 & 1.00 & 0.00 & 1.00 & 0.00 & 1.00 \\
\hline 277.0 & 1.91 & 1.99 & I & 1.00 & 0.00 & 1.00 & 0.00 & 1.00 & 0.00 \\
\hline 277.0 & 2.54 & 2.38 & I & 0.85 & 0.15 & 0.98 & 0.02 & 0.98 & 0.02 \\
\hline 280.0 & 2.74 & 2.86 & $\mathrm{I}$ & 1.00 & 0.00 & 1.00 & 0.00 & 1.00 & 0.00 \\
\hline 280.0 & 3.52 & 3.57 & $\mathrm{I}$ & 0.83 & 0.17 & 0.98 & 0.02 & 0.98 & 0.02 \\
\hline 280.0 & 4.14 & 4.35 & I & 0.70 & 0.30 & 0.96 & 0.04 & 0.95 & 0.05 \\
\hline 280.0 & 4.95 & 5.29 & $\mathrm{I}$ & 0.59 & 0.41 & 0.94 & 0.06 & 0.92 & 0.08 \\
\hline 280.0 & 8.09 & 8.50 & $\mathrm{I}$ & 0.39 & 0.61 & 0.86 & 0.14 & 0.83 & 0.17 \\
\hline 280.0 & 14.64 & 13.50 & I & 0.25 & 0.75 & 0.64 & 0.36 & 0.68 & 0.32 \\
\hline 280.0 & 20.29 & 18.39 & $\mathrm{I}$ & 0.17 & 0.83 & 0.45 & 0.55 & 0.55 & 0.45 \\
\hline 280.0 & 26.09 & 25.74 & I & 0.09 & 0.91 & 0.22 & 0.78 & 0.35 & 0.65 \\
\hline 280.0 & 31.58 & 38.81 & $\mathrm{I}$ & 0.00 & 1.00 & 0.00 & 1.00 & 0.00 & 1.00 \\
\hline \multicolumn{2}{|c|}{ AADp } & $6.5 \%$ & \multicolumn{5}{|c|}{ AADc } & \multicolumn{2}{|c|}{0.04} \\
\hline
\end{tabular}

229 As Figure 3 and Table 6 indicate, the thermodynamic model acceptably predicted the equilibrium 230 pressure (average deviation 6.5\%). The hydrate composition simulation could be categorized in 231 two parts. The first part is when carbon dioxide was dominant in the hydrate phase. In this case, 232 the simulation results were well predicated (average absolute deviation for 13 equilibrium points 233 was 0.016 mole fraction). While nitrogen was dominant in the hydrate phase, the average 234 absolute deviation was 0.071 in mole fraction.

\section{4.2. Material balance and volumetric properties evaluated from equation of state}

236 Ohgaki et al. investigated the phase equilibrium of carbon dioxide-methane hydrate at $280.3 \mathrm{~K}$ 237 [65]. They obtained the guest composition in gas, liquid and hydrate phases at isothermal 238 conditions in a batch reactor. Their experimental procedure is briefly described as follows: They 239 injected separately carbon dioxide and methane to the reactor. Pure water was then introduced to 240 the reactor. The amount of each material was weighed. Thanks to a gas chromatograph, they 241 determined the gas composition of carbon dioxide and methane at equilibrium temperature and 242 pressure. The solubility of carbon dioxide and methane in water was calculated based on Henry 243 constants. They assumed that the general formula for mixed carbon dioxide-methane hydrate is as 244 follows:

$245 z \mathrm{CO}_{2} \cdot(1-z) \cdot q \mathrm{H}_{2} \mathrm{O}$

246 where $\mathrm{z}$ is the mole fraction of carbon dioxide in hydrate phase and $\mathrm{q}$ is the hydration number at 247 ideal condition. They also hypothesized that the molar volume of hydrate is $130.1 \mathrm{~cm}^{3} / \mathrm{mol}^{\text {[65] }}$. 248 They calculated the volumetric properties from Soave-Redlich-Kwong equation of state or 
IUPAC recommended equation of state for carbon dioxide and methane [60,65-67]. The hydrate compositions were then calculated by the material balance.

251 Figure 4 shows their experimental results for hydrate phase composition and our simulation 252 results. Furthermore, Table 7 summarizes the experimental and modeling results by details for 253 carbon dioxide-methane hydrate at $280.3 \mathrm{~K}$.

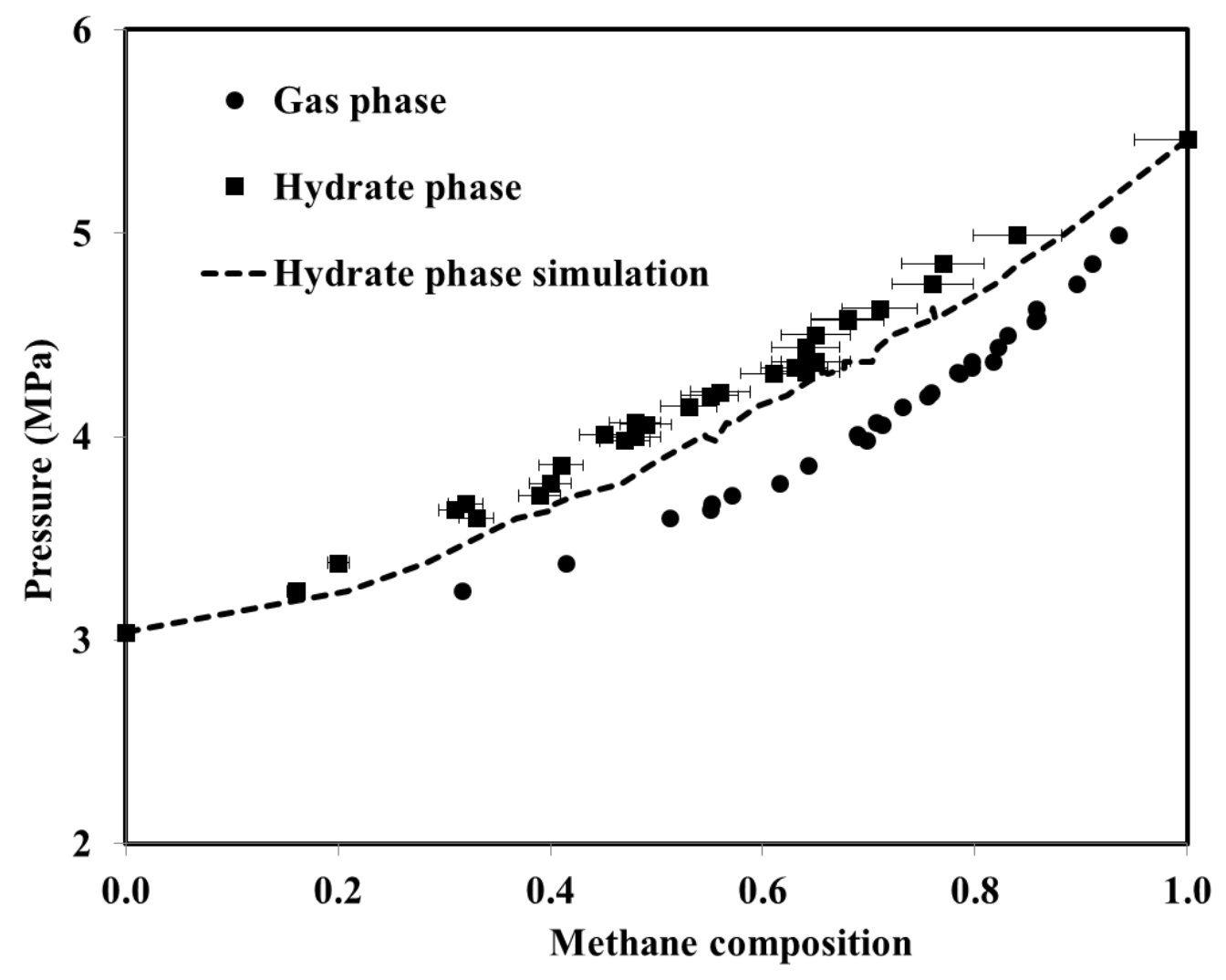

Figure 4. Carbon dioxide-methane hydrates phase equilibria at $280.3 \mathrm{~K}$. Experimental [65] and simulation results (sI). Error bars correspond to the standard uncertainty of experimental hydrate composition.

Table 7. Experimental results of Ohgaki et al. [65] for methane-carbon dioxide mixture hydrates at $280.3 \mathrm{~K}$ and simulation results.

\begin{tabular}{|c|c|c|c|c|c|c|c|c|}
\hline \multirow{2}{*}{$\begin{array}{c}\text { Pexp } \\
(\mathrm{MPa}) \\
( \pm 0.005)\end{array}$} & \multirow{2}{*}{$\begin{array}{c}\text { Ppre } \\
(\mathrm{MPa})\end{array}$} & \multirow{2}{*}{$\mathrm{S}$} & \multicolumn{2}{|c|}{ Gas composition (exp) } & \multicolumn{2}{|c|}{$\begin{array}{c}\text { Hydrate composition } \\
(\exp )( \pm 5 \%)\end{array}$} & \multicolumn{2}{|c|}{$\begin{array}{c}\text { Hydrate composition } \\
\text { (pre) }\end{array}$} \\
\cline { 5 - 9 } & & & $\mathrm{CO}_{2}$ & $\mathrm{CH}_{4}$ & $\mathrm{CO}_{2}$ & $\mathrm{CH}_{4}$ & $\mathrm{CO}_{2}$ & $\mathrm{CH}_{4}$ \\
\hline 3.04 & 2.98 & $\mathrm{I}$ & 1.00 & 0.00 & 1.00 & 0.00 & 1.00 & 0.00 \\
\hline 3.24 & 3.46 & $\mathrm{I}$ & 0.68 & 0.32 & 0.84 & 0.16 & 0.79 & 0.21 \\
\hline 3.38 & 3.55 & $\mathrm{I}$ & 0.59 & 0.42 & 0.80 & 0.20 & 0.72 & 0.28 \\
\hline 3.60 & 3.85 & $\mathrm{I}$ & 0.49 & 0.51 & 0.67 & 0.33 & 0.63 & 0.37 \\
\hline 3.64 & 3.93 & $\mathrm{I}$ & 0.45 & 0.55 & 0.69 & 0.31 & 0.60 & 0.40 \\
\hline 3.67 & 3.93 & $\mathrm{I}$ & 0.45 & 0.55 & 0.68 & 0.32 & 0.60 & 0.40 \\
\hline 3.71 & 3.98 & $\mathrm{I}$ & 0.43 & 0.57 & 0.61 & 0.39 & 0.58 & 0.42 \\
\hline
\end{tabular}




\begin{tabular}{|l|l|l|l|l|l|l|l|l|}
\hline 3.77 & 4.09 & $\mathrm{I}$ & 0.38 & 0.62 & 0.60 & 0.40 & 0.53 & 0.47 \\
\hline 3.86 & 4.16 & $\mathrm{I}$ & 0.36 & 0.64 & 0.59 & 0.41 & 0.50 & 0.50 \\
\hline 4.22 & 4.49 & $\mathrm{I}$ & 0.24 & 0.76 & 0.44 & 0.56 & 0.37 & 0.63 \\
\hline 4.31 & 4.57 & $\mathrm{I}$ & 0.22 & 0.79 & 0.39 & 0.61 & 0.34 & 0.66 \\
\hline 4.32 & 4.57 & $\mathrm{I}$ & 0.22 & 0.78 & 0.36 & 0.64 & 0.34 & 0.66 \\
\hline 4.34 & 4.61 & $\mathrm{I}$ & 0.20 & 0.80 & 0.37 & 0.63 & 0.32 & 0.68 \\
\hline 4.37 & 4.61 & $\mathrm{I}$ & 0.20 & 0.80 & 0.35 & 0.65 & 0.32 & 0.68 \\
\hline 4.37 & 4.68 & $\mathrm{I}$ & 0.18 & 0.82 & 0.36 & 0.64 & 0.30 & 0.70 \\
\hline 4.44 & 4.70 & $\mathrm{I}$ & 0.18 & 0.82 & 0.36 & 0.64 & 0.29 & 0.71 \\
\hline 4.50 & 4.73 & $\mathrm{I}$ & 0.17 & 0.83 & 0.35 & 0.65 & 0.28 & 0.72 \\
\hline 4.57 & 4.82 & $\mathrm{I}$ & 0.14 & 0.86 & 0.32 & 0.68 & 0.24 & 0.76 \\
\hline 3.98 & 4.31 & $\mathrm{I}$ & 0.30 & 0.70 & 0.53 & 0.47 & 0.44 & 0.56 \\
\hline 4.00 & 4.29 & $\mathrm{I}$ & 0.31 & 0.69 & 0.52 & 0.48 & 0.45 & 0.55 \\
\hline 4.01 & 4.28 & $\mathrm{I}$ & 0.31 & 0.69 & 0.55 & 0.45 & 0.45 & 0.55 \\
\hline 4.06 & 4.35 & $\mathrm{I}$ & 0.29 & 0.71 & 0.51 & 0.49 & 0.43 & 0.57 \\
\hline 4.07 & 4.33 & $\mathrm{I}$ & 0.29 & 0.71 & 0.52 & 0.48 & 0.43 & 0.57 \\
\hline 4.15 & 4.41 & $\mathrm{I}$ & 0.27 & 0.73 & 0.47 & 0.53 & 0.41 & 0.59 \\
\hline 4.20 & 4.48 & $\mathrm{I}$ & 0.25 & 0.76 & 0.45 & 0.55 & 0.38 & 0.62 \\
\hline 4.58 & 4.83 & $\mathrm{I}$ & 0.14 & 0.86 & 0.32 & 0.68 & 0.24 & 0.76 \\
\hline 4.63 & 4.83 & $\mathrm{I}$ & 0.14 & 0.86 & 0.29 & 0.71 & 0.24 & 0.76 \\
\hline 4.75 & 4.96 & $\mathrm{I}$ & 0.10 & 0.90 & 0.24 & 0.76 & 0.18 & 0.82 \\
\hline 4.85 & 5.03 & $\mathrm{I}$ & 0.09 & 0.91 & 0.23 & 0.77 & 0.16 & 0.84 \\
\hline 4.99 & 5.14 & $\mathrm{I}$ & 0.07 & 0.94 & 0.16 & 0.84 & 0.12 & 0.88 \\
\hline 5.46 & 5.44 & $\mathrm{I}$ & 0.00 & 1.00 & 0.00 & 1.00 & 0.00 & 1.00 \\
\hline $\mathrm{AADp}$ & $5.8 \%$ & & & $\mathrm{AADc}$ & & & & 0.06 \\
\hline & & & & & & & & \\
\hline
\end{tabular}

260 They observed that at each equilibrium condition, the mole fraction of carbon dioxide in hydrate 261 phase is significantly larger than in gas phase. Hence, based on the idea of methane exploration 262 by carbon dioxide injection and by defining the average distribution coefficient of methane, they 263 concluded that methane in hydrate phase can be replaced by carbon dioxide; as a result, methane 264 concentration in the gas phase will be increased.

265 The simulation results agreed well with the experimental data for hydrate equilibrium pressure 266 (AADp 5.8\%). Additionally, the mole fraction deviation of hydrate composition calculations was 267 about 0.06.

268 Belandria et al. investigated the compositional analysis of carbon dioxide-methane hydrate by the 269 same method as Ohgaki et al. [65,68]. Our simulation results based on the experimental data of 270 Belandria et al. are presented in Table 8 and Figure 5. As it is clear on the table, the 271 thermodynamic model predicted hydrate equilibrium pressure with an acceptable error (7.8\%). 272 Although the hydrate composition simulation had mostly an adequate agreement with the data 
273 obtained, several experimental equilibrium points were poorly simulated (for instance $280 \mathrm{~K}$ and $2743.54 \mathrm{MPa}$ ). Additionally, they reported that CSMGem model was not capable to converge three 275 phase flash calculations in some cases, in comparison, the thermodynamic model had no problem 276 with three phase flash calculations [68].

Table 8. Experimental results of Belandria et al. [68] for methane-carbon dioxide hydrates and simulation results.

\begin{tabular}{|c|c|c|c|c|c|c|c|c|c|}
\hline \multirow{2}{*}{$\begin{array}{l}\mathrm{T}(\mathrm{K}) \\
( \pm 0.02)\end{array}$} & \multirow{2}{*}{$\begin{array}{c}\text { Pexp } \\
(\mathrm{MPa}) \\
( \pm 0.002)\end{array}$} & \multirow{2}{*}{$\begin{array}{l}\text { Ppre } \\
(\mathrm{MPa})\end{array}$} & \multirow[t]{2}{*}{$S$} & \multicolumn{2}{|c|}{$\begin{array}{c}\text { Gas composition } \\
\text { (exp) }\end{array}$} & \multicolumn{2}{|c|}{$\begin{array}{l}\text { Hydrate composition } \\
\text { (exp) }( \pm 1 \%)\end{array}$} & \multicolumn{2}{|c|}{$\begin{array}{l}\text { Hydrate composition } \\
\text { (pre) }\end{array}$} \\
\hline & & & & $\mathrm{CO}_{2}$ & $\mathrm{CH}_{4}$ & $\mathrm{CO}_{2}$ & $\mathrm{CH}_{4}$ & $\mathrm{CO}_{2}$ & $\mathrm{CH}_{4}$ \\
\hline 273.6 & 2.44 & 2.55 & $\mathrm{I}$ & 0.08 & 0.92 & 0.10 & 0.90 & 0.16 & 0.84 \\
\hline 273.6 & 1.84 & 2.03 & I & 0.35 & 0.66 & 0.55 & 0.45 & 0.52 & 0.48 \\
\hline 273.6 & 1.94 & 2.12 & $\mathrm{I}$ & 0.29 & 0.71 & 0.39 & 0.61 & 0.46 & 0.54 \\
\hline 273.6 & 2.05 & 2.24 & I & 0.22 & 0.78 & 0.29 & 0.71 & 0.37 & 0.63 \\
\hline 273.6 & 1.51 & 1.67 & I & 0.63 & 0.37 & 0.88 & 0.12 & 0.77 & 0.23 \\
\hline 273.6 & 1.61 & 1.77 & I & 0.55 & 0.46 & 0.80 & 0.20 & 0.71 & 0.29 \\
\hline 275.2 & 2.58 & 2.77 & $\mathrm{I}$ & 0.17 & 0.83 & 0.34 & 0.66 & 0.29 & 0.71 \\
\hline 275.2 & 2.77 & 2.98 & I & 0.09 & 0.91 & 0.18 & 0.82 & 0.16 & 0.84 \\
\hline 275.2 & 2.12 & 2.33 & $\mathrm{I}$ & 0.38 & 0.62 & 0.65 & 0.35 & 0.56 & 0.44 \\
\hline 275.2 & 2.22 & 2.48 & I & 0.30 & 0.70 & 0.59 & 0.41 & 0.47 & 0.53 \\
\hline 275.2 & 2.40 & 2.63 & I & 0.23 & 0.77 & 0.37 & 0.63 & 0.38 & 0.62 \\
\hline 275.2 & 1.79 & 1.96 & I & 0.66 & 0.34 & 0.83 & 0.17 & 0.79 & 0.21 \\
\hline 275.2 & 1.87 & 2.07 & I & 0.57 & 0.44 & 0.75 & 0.25 & 0.72 & 0.28 \\
\hline 276.1 & 2.81 & 3.00 & I & 0.18 & 0.82 & 0.26 & 0.74 & 0.31 & 0.69 \\
\hline 276.1 & 3.03 & 3.12 & I & 0.13 & 0.87 & 0.24 & 0.76 & 0.24 & 0.76 \\
\hline 276.1 & 3.03 & 3.23 & I & 0.10 & 0.90 & 0.24 & 0.76 & 0.18 & 0.82 \\
\hline 276.1 & 2.32 & 2.43 & I & 0.41 & 0.60 & 0.64 & 0.36 & 0.57 & 0.43 \\
\hline 276.1 & 2.50 & 2.70 & $\mathrm{I}$ & 0.32 & 0.69 & 0.40 & 0.60 & 0.48 & 0.52 \\
\hline 276.1 & 2.69 & 2.88 & I & 0.23 & 0.77 & 0.31 & 0.69 & 0.38 & 0.62 \\
\hline 276.1 & 1.99 & 2.14 & $\mathrm{I}$ & 0.67 & 0.33 & 0.88 & 0.12 & 0.80 & 0.20 \\
\hline 276.1 & 2.17 & 2.26 & I & 0.58 & 0.42 & 0.78 & 0.22 & 0.73 & 0.27 \\
\hline 278.1 & 3.42 & 3.64 & $\mathrm{I}$ & 0.20 & 0.80 & 0.23 & 0.77 & 0.33 & 0.67 \\
\hline 278.1 & 3.63 & 3.83 & I & 0.14 & 0.86 & 0.23 & 0.78 & 0.24 & 0.76 \\
\hline 278.1 & 3.80 & 3.95 & $\mathrm{I}$ & 0.10 & 0.90 & 0.15 & 0.85 & 0.19 & 0.81 \\
\hline 278.1 & 3.04 & 3.33 & I & 0.32 & 0.68 & 0.46 & 0.54 & 0.48 & 0.52 \\
\hline 278.1 & 3.32 & 3.55 & I & 0.23 & 0.77 & 0.27 & 0.73 & 0.37 & 0.63 \\
\hline 278.1 & 2.58 & 2.77 & I & 0.61 & 0.39 & 0.79 & 0.21 & 0.74 & 0.26 \\
\hline 279.2 & 3.57 & 4.09 & I & 0.20 & 0.80 & 0.27 & 0.73 & 0.33 & 0.67 \\
\hline 280.2 & 4.49 & 4.76 & I & 0.15 & 0.85 & 0.31 & 0.69 & 0.25 & 0.75 \\
\hline 280.2 & 4.66 & 4.90 & $\mathrm{I}$ & 0.11 & 0.89 & 0.25 & 0.76 & 0.19 & 0.81 \\
\hline 280.2 & 3.54 & 4.14 & I & 0.34 & 0.66 & 0.73 & 0.27 & 0.49 & 0.51 \\
\hline 280.2 & 4.11 & 4.46 & I & 0.24 & 0.77 & 0.34 & 0.66 & 0.37 & 0.63 \\
\hline
\end{tabular}




\begin{tabular}{|l|l|l|l|l|l|l|l|l|l|}
\hline 280.2 & 3.14 & 3.53 & $\mathrm{I}$ & 0.62 & 0.38 & 0.86 & 0.14 & 0.74 & 0.26 \\
\hline 280.2 & 3.48 & 3.75 & $\mathrm{I}$ & 0.49 & 0.51 & 0.79 & 0.21 & 0.64 & 0.36 \\
\hline 282.2 & 5.77 & 6.08 & $\mathrm{I}$ & 0.11 & 0.89 & 0.28 & 0.72 & 0.19 & 0.81 \\
\hline 284.2 & 7.19 & 7.65 & $\mathrm{I}$ & 0.12 & 0.89 & 0.11 & 0.89 & 0.18 & 0.82 \\
\hline \multicolumn{7}{|c|}{ AADp } & $7.8 \%$ & \multicolumn{7}{|c|}{ AADc } \\
\hline
\end{tabular}

279

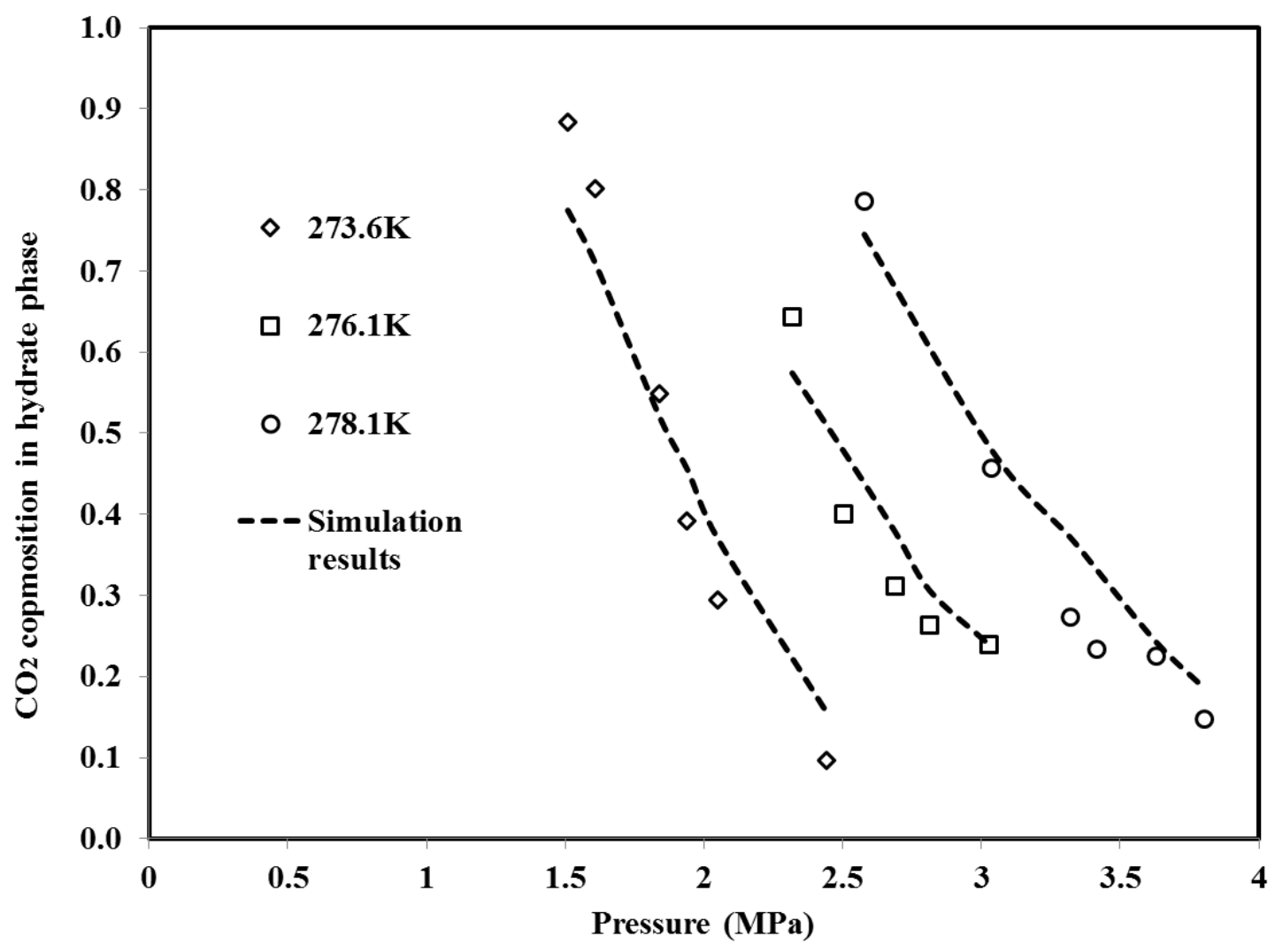

Figure 5. Hydrate composition for methane-carbon dioxide hydrates. Experimental data [68] and simulation results (sI).

Belandria et al. also studied the hydrate composition of carbon dioxide-nitrogen mixture using the same method $[65,69]$. They calculated the guest composition in gas, liquid and hydrate phases based on the material balance and volumetric properties evaluated from equation of state. Table 9 details their experimental data and our simulation results. Moreover, Figure 6 illustrates the experimental [69] and simulation results for two isotherms of $\mathrm{N}_{2} / \mathrm{CO}_{2}$ binary hydrates.

Table 9. Experimental results of Belandria et al. [69] for nitrogen-carbon dioxide mixture hydrates and simulation results.

\begin{tabular}{|c|c|c|c|c|c|c|c|c|c|}
\hline \multirow{2}{*}{$\begin{array}{c}\mathrm{T}(\mathrm{K}) \\
( \pm 0.02)\end{array}$} & $\begin{array}{c}\text { Pexp } \\
(\mathrm{MPa}) \\
( \pm 0.002)\end{array}$ & $\begin{array}{c}\text { Ppre } \\
(\mathrm{MPa})\end{array}$ & \multirow{2}{*}{$\mathrm{S}$} & \multicolumn{2}{|c|}{$\begin{array}{c}\text { Gas composition } \\
(\exp )\end{array}$} & \multicolumn{2}{c|}{$\begin{array}{c}\text { Hydrate composition } \\
(\exp )( \pm 1 \%)\end{array}$} & \multicolumn{2}{c|}{$\begin{array}{c}\text { Hydrate composition } \\
(\text { pre })\end{array}$} \\
\cline { 5 - 10 } & & & $\mathrm{CO}_{2}$ & $\mathrm{~N}_{2}$ & $\mathrm{CO}_{2}$ & $\mathrm{~N}_{2}$ & $\mathrm{CO}_{2}$ & $\mathrm{~N}_{2}$ \\
\hline 273.6 & 2.03 & 2.22 & $\mathrm{I}$ & 0.62 & 0.38 & 0.97 & 0.03 & 0.95 & 0.05 \\
\hline
\end{tabular}




\begin{tabular}{|c|c|c|c|c|c|c|c|c|c|}
\hline 273.6 & 8.15 & 7.22 & I & 0.17 & 0.83 & 0.66 & 0.34 & 0.66 & 0.34 \\
\hline 273.6 & 11.94 & 6.94 & I & 0.18 & 0.82 & 0.37 & 0.63 & 0.67 & 0.33 \\
\hline 273.6 & 2.96 & 3.17 & I & 0.43 & 0.57 & 0.90 & 0.10 & 0.89 & 0.11 \\
\hline 274.6 & 2.54 & 2.10 & I & 0.73 & 0.27 & 0.74 & 0.26 & 0.97 & 0.03 \\
\hline 274.9 & 5.20 & 2.21 & I & 0.72 & 0.28 & 0.79 & 0.21 & 0.96 & 0.04 \\
\hline 275.2 & 2.29 & 2.51 & I & 0.66 & 0.34 & 0.90 & 0.10 & 0.95 & 0.05 \\
\hline 275.2 & 2.64 & 2.25 & I & 0.73 & 0.27 & 0.89 & 0.11 & 0.97 & 0.03 \\
\hline 275.2 & 3.26 & 3.69 & I & 0.45 & 0.55 & 0.88 & 0.12 & 0.89 & 0.11 \\
\hline 275.2 & 7.45 & 8.83 & I & 0.17 & 0.83 & 0.82 & 0.18 & 0.64 & 0.36 \\
\hline 275.2 & 8.25 & 8.74 & I & 0.18 & 0.82 & 0.80 & 0.20 & 0.65 & 0.35 \\
\hline 275.2 & 12.75 & 9.32 & I & 0.16 & 0.84 & 0.38 & 0.62 & 0.62 & 0.38 \\
\hline 275.6 & 2.71 & 2.36 & $\mathrm{I}$ & 0.73 & 0.27 & 0.76 & 0.24 & 0.97 & 0.03 \\
\hline 275.8 & 5.38 & 2.45 & I & 0.72 & 0.28 & 0.80 & 0.20 & 0.96 & 0.04 \\
\hline 276.1 & 2.50 & 2.69 & $\mathrm{I}$ & 0.68 & 0.32 & 0.98 & 0.02 & 0.96 & 0.04 \\
\hline 276.1 & 2.87 & 2.50 & I & 0.73 & 0.27 & 0.79 & 0.21 & 0.97 & 0.03 \\
\hline 276.1 & 3.70 & 3.80 & I & 0.49 & 0.51 & 0.70 & 0.30 & 0.90 & 0.10 \\
\hline 276.1 & 4.40 & 4.70 & $\mathrm{I}$ & 0.40 & 0.60 & 0.69 & 0.31 & 0.86 & 0.14 \\
\hline 276.1 & 8.58 & 9.07 & I & 0.20 & 0.80 & 0.57 & 0.43 & 0.67 & 0.33 \\
\hline 276.7 & 3.70 & 4.11 & I & 0.49 & 0.51 & 0.70 & 0.30 & 0.90 & 0.10 \\
\hline 277.1 & 2.71 & 2.94 & I & 0.71 & 0.30 & 0.84 & 0.16 & 0.96 & 0.04 \\
\hline 277.3 & 3.13 & 2.89 & I & 0.73 & 0.27 & 0.83 & 0.17 & 0.96 & 0.04 \\
\hline 277.8 & 6.16 & 3.01 & I & 0.75 & 0.25 & 0.86 & 0.14 & 0.97 & 0.03 \\
\hline 278.1 & 2.97 & 3.21 & I & 0.73 & 0.27 & 0.89 & 0.11 & 0.96 & 0.04 \\
\hline 278.1 & 3.41 & 3.19 & I & 0.73 & 0.27 & 0.75 & 0.25 & 0.96 & 0.04 \\
\hline 278.1 & 4.19 & 4.63 & $\mathrm{I}$ & 0.52 & 0.48 & 0.66 & 0.35 & 0.91 & 0.09 \\
\hline 278.1 & 9.15 & 10.69 & I & 0.23 & 0.77 & 0.54 & 0.46 & 0.69 & 0.31 \\
\hline 278.1 & 14.26 & 16.69 & I & 0.13 & 0.87 & 0.51 & 0.49 & 0.49 & 0.51 \\
\hline 279.7 & 4.82 & 5.42 & I & 0.56 & 0.44 & 0.70 & 0.30 & 0.91 & 0.09 \\
\hline 279.7 & 10.02 & 12.26 & $\mathrm{I}$ & 0.26 & 0.74 & 0.61 & 0.39 & 0.71 & 0.29 \\
\hline 279.7 & 15.82 & 19.25 & I & 0.15 & 0.85 & 0.55 & 0.45 & 0.50 & 0.50 \\
\hline 281.2 & 17.63 & 21.78 & $\mathrm{I}$ & 0.18 & 0.82 & 0.58 & 0.42 & 0.53 & 0.47 \\
\hline 281.7 & 6.33 & 5.22 & I & 0.75 & 0.25 & 0.81 & 0.19 & 0.96 & 0.04 \\
\hline \multicolumn{2}{|c|}{$\mathrm{AADp}$} & $17.4 \%$ & \multicolumn{5}{|c|}{$\mathrm{AADc}$} & \multicolumn{2}{|c|}{0.13} \\
\hline
\end{tabular}

290

291 From Table 9 and Figure 6, notice that neither equilibrium pressure nor hydrate composition were 292 well simulated. Nevertheless, at equilibrium pressures below $7 \mathrm{MPa}$, the simulation results were 293 satisfactory. In contrast, equilibrium pressures more than $7 \mathrm{MPa}$ were poorly simulated. In the 294 case of hydrate composition, the average deviation was large (0.13) compared to the simulation 295 of experimental data of Kang et al. (0.04) for the same mixture. This divergence might be due to 296 different experimental procedures. 


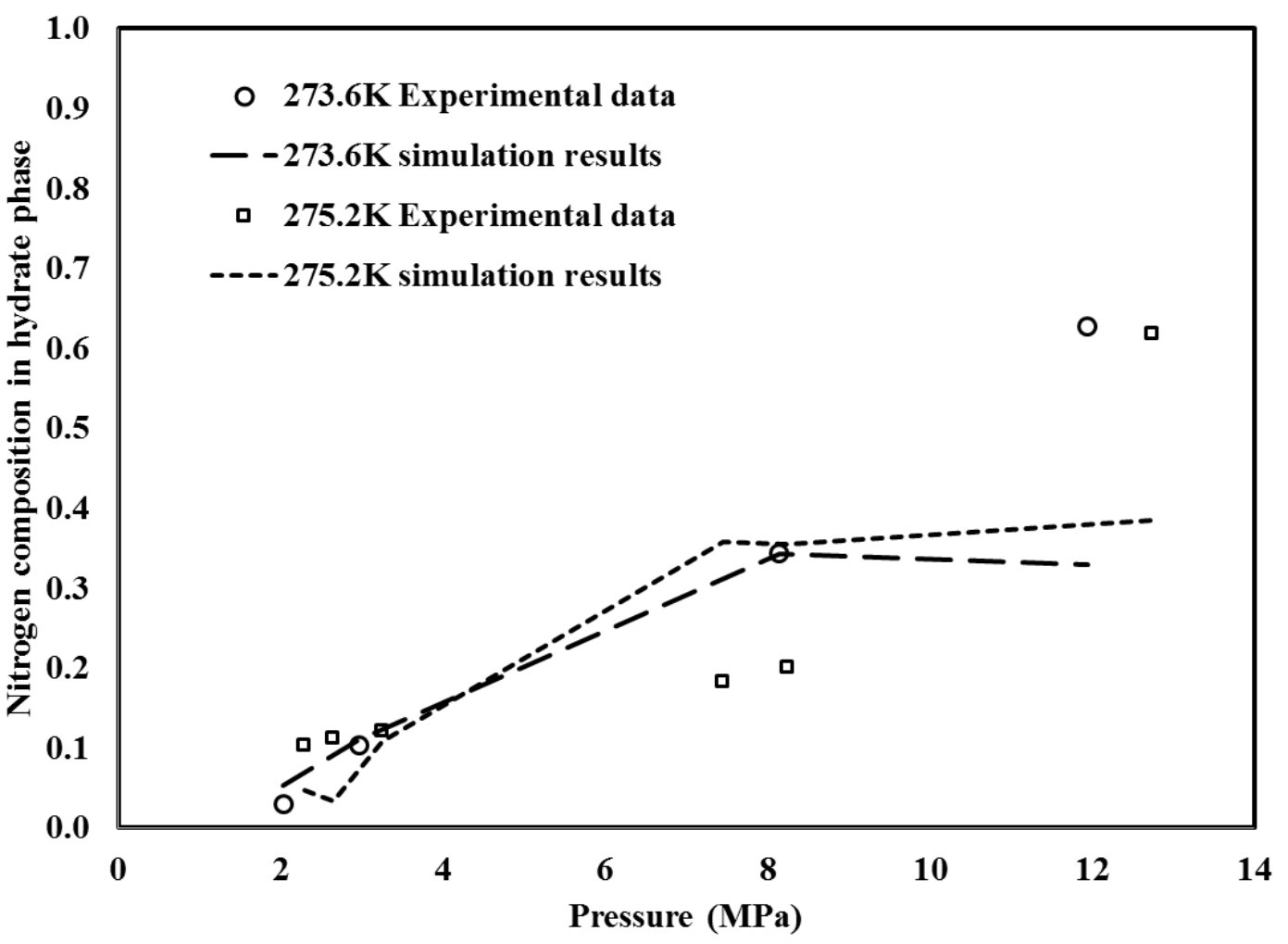

Figure 6. Hydrate composition for nitrogen-carbon dioxide hydrates at two isotherms. Experimental data [69] and simulation results (sI).

\subsection{Gas uptake at isobaric equilibrium condition}

302 Seo et al. studied the vapor-liquid-hydrate equilibrium conditions of nitrogen-carbon dioxide and 303 methane-carbon dioxide mixtures at isobaric condition [70]. They performed their experiments in 304 a batch reactor by injecting gas mixtures and water by a syringe pump at a desired pressure. Then 305 they decreased the temperature to 5 degrees Kelvin below the hydrate formation temperature. 306 Hydrate formation led to a decrease in hydrate pressure. In order to keep constant the pressure, 307 they recharged reactor by the gas mixtures. Then, they increased the temperature by the rate of 1$3082 \mathrm{~K}$ per hour. The pressure increased due to hydrate dissociation. Then, the dissociated gases 309 were vented. When only a small amount of hydrate crystals remained in the cell and the pressure 310 was constant, this was considered as the three phase equilibrium [70]. Both their experimental 311 and our simulation results for nitrogen-carbon dioxide mixture are listed and presented in Table 31210 and Figure 7. Moreover, the results of methane-carbon dioxide mixture are presented in Table 31311. 


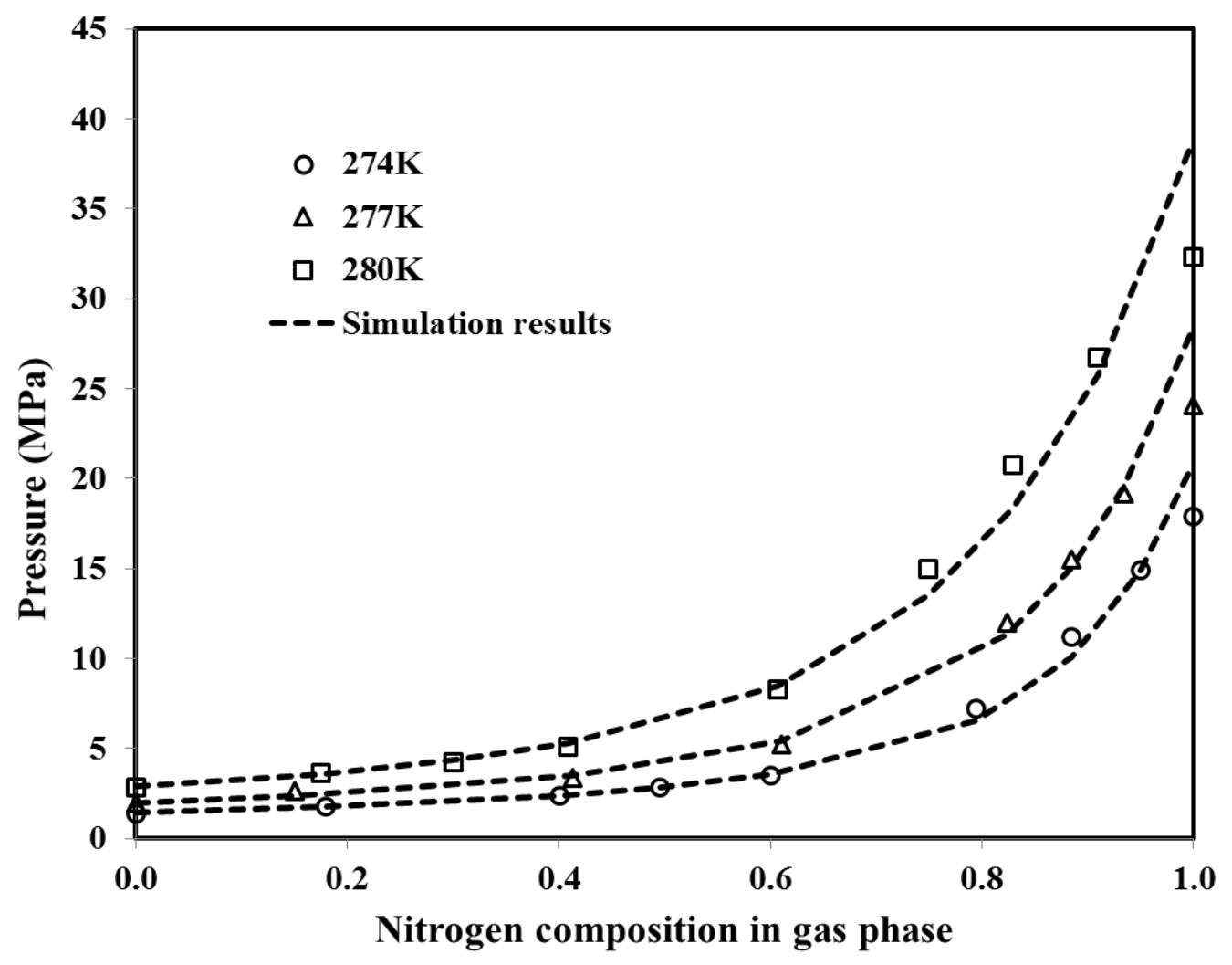

Figure 7. Pressure-gas composition diagram for nitrogen-carbon dioxide at three isotherms (sI). Experimental data from Seo et al. [70] and our prediction results

Table 10. Experimental results of carbon dioxide-nitrogen hydrate equilibrium pressure and composition from Seo et al. [70] and our simulation results. Composition uncertainties were not provided.

\begin{tabular}{|c|c|c|c|c|c|c|c|c|c|}
\hline \multirow{2}{*}{$\begin{array}{c}\mathrm{T}(\mathrm{K}) \\
( \pm 0.1)\end{array}$} & $\begin{array}{c}\text { Pexp } \\
(\mathrm{MPa}) \\
( \pm 0.01)\end{array}$ & \multirow{2}{*}{$\begin{array}{c}\text { Ppre } \\
(\mathrm{MPa})\end{array}$} & $\mathrm{S}$ & \multicolumn{2}{|c|}{$\begin{array}{c}\text { Gas composition } \\
(\mathrm{exp})\end{array}$} & \multicolumn{2}{c|}{$\begin{array}{c}\text { Hydrate composition } \\
(\mathrm{exp})\end{array}$} & \multicolumn{2}{c|}{$\begin{array}{c}\text { Hydrate composition } \\
\text { (pre) }\end{array}$} \\
\cline { 5 - 10 } & & $\mathrm{CO}_{2}$ & $\mathrm{~N}_{2}$ & $\mathrm{CO}_{2}$ & $\mathrm{~N}_{2}$ & $\mathrm{CO}_{2}$ & $\mathrm{~N}_{2}$ \\
\hline 274 & 1.39 & 1.42 & $\mathrm{I}$ & 1.00 & 0.00 & 1.00 & 0.00 & 1.00 & 0.00 \\
\hline 274 & 1.77 & 1.74 & $\mathrm{I}$ & 0.82 & 0.18 & 0.99 & 0.02 & 0.98 & 0.02 \\
\hline 274 & 2.35 & 2.39 & $\mathrm{I}$ & 0.60 & 0.40 & 0.95 & 0.05 & 0.94 & 0.06 \\
\hline 274 & 2.84 & 2.84 & $\mathrm{I}$ & 0.50 & 0.50 & 0.93 & 0.07 & 0.92 & 0.08 \\
\hline 274 & 3.46 & 3.57 & $\mathrm{I}$ & 0.40 & 0.60 & 0.90 & 0.10 & 0.87 & 0.13 \\
\hline 274 & 7.24 & 6.55 & $\mathrm{I}$ & 0.21 & 0.79 & 0.58 & 0.42 & 0.71 & 0.29 \\
\hline 274 & 11.20 & 10.03 & $\mathrm{I}$ & 0.12 & 0.88 & 0.34 & 0.66 & 0.53 & 0.47 \\
\hline 274 & 14.93 & 14.91 & $\mathrm{I}$ & 0.05 & 0.95 & 0.18 & 0.82 & 0.28 & 0.72 \\
\hline 274 & 17.93 & 20.81 & $\mathrm{I}$ & 0.00 & 1.00 & 0.00 & 1.00 & 0.00 & 1.00 \\
\hline 277 & 1.95 & 1.99 & $\mathrm{I}$ & 1.00 & 0.00 & 1.00 & 0.00 & 1.00 & 0.00 \\
\hline 277 & 2.60 & 2.38 & $\mathrm{I}$ & 0.85 & 0.15 & 0.98 & 0.02 & 0.98 & 0.02 \\
\hline 277 & 3.38 & 3.52 & $\mathrm{I}$ & 0.59 & 0.41 & 0.95 & 0.05 & 0.93 & 0.07 \\
\hline 277 & 5.23 & 5.40 & $\mathrm{I}$ & 0.39 & 0.61 & 0.89 & 0.11 & 0.85 & 0.15 \\
\hline 277 & 11.98 & 11.30 & $\mathrm{I}$ & 0.18 & 0.82 & 0.54 & 0.46 & 0.62 & 0.38 \\
\hline
\end{tabular}




\begin{tabular}{|l|c|c|c|c|c|c|c|c|c|}
\hline 277 & 15.50 & 15.07 & $\mathrm{I}$ & 0.12 & 0.88 & 0.35 & 0.65 & 0.48 & 0.52 \\
\hline 277 & 19.17 & 19.56 & $\mathrm{I}$ & 0.07 & 0.93 & 0.19 & 0.81 & 0.31 & 0.69 \\
\hline 277 & 24.04 & 28.37 & $\mathrm{I}$ & 0.00 & 1.00 & 0.00 & 1.00 & 0.00 & 1.00 \\
\hline 280 & 2.80 & 2.86 & $\mathrm{I}$ & 1.00 & 0.00 & 1.00 & 0.00 & 1.00 & 0.00 \\
\hline 280 & 3.60 & 3.59 & $\mathrm{I}$ & 0.83 & 0.18 & 0.98 & 0.02 & 0.98 & 0.02 \\
\hline 280 & 4.23 & 4.35 & $\mathrm{I}$ & 0.70 & 0.30 & 0.96 & 0.04 & 0.95 & 0.05 \\
\hline 280 & 5.07 & 5.29 & $\mathrm{I}$ & 0.59 & 0.41 & 0.94 & 0.06 & 0.92 & 0.08 \\
\hline 280 & 8.28 & 8.50 & $\mathrm{I}$ & 0.39 & 0.61 & 0.86 & 0.14 & 0.83 & 0.17 \\
\hline 280 & 14.97 & 13.50 & $\mathrm{I}$ & 0.25 & 0.75 & 0.64 & 0.36 & 0.68 & 0.32 \\
\hline 280 & 20.75 & 18.39 & $\mathrm{I}$ & 0.17 & 0.83 & 0.45 & 0.55 & 0.55 & 0.45 \\
\hline 280 & 26.69 & 25.74 & $\mathrm{I}$ & 0.09 & 0.91 & 0.22 & 0.78 & 0.35 & 0.65 \\
\hline 280 & 32.31 & 38.81 & $\mathrm{I}$ & 0.00 & 1.00 & 0.00 & 1.00 & 0.00 & 1.00 \\
\hline \multicolumn{7}{|c|}{ AADp } & $5.7 \%$ & \multicolumn{7}{|c|}{ AADc } & & & 0.04 \\
\hline
\end{tabular}

Table 11. Experimental results of carbon dioxide-methane hydrate equilibrium pressure and composition from Seo et al. [70] and our simulation results. Composition uncertainties were not provided.

\begin{tabular}{|c|c|c|c|c|c|c|c|c|c|}
\hline \multirow{2}{*}{$\begin{array}{l}\mathrm{T}(\mathrm{K}) \\
( \pm 0.1)\end{array}$} & \multirow{2}{*}{$\begin{array}{c}\text { Pexp } \\
(\mathrm{MPa}) \\
( \pm 0.01) \\
\end{array}$} & \multirow{2}{*}{$\begin{array}{l}\text { Ppre } \\
(\mathrm{MPa})\end{array}$} & \multirow{2}{*}{$S$} & \multicolumn{2}{|c|}{$\begin{array}{c}\text { Gas composition } \\
\text { (exp) }\end{array}$} & \multicolumn{2}{|c|}{$\begin{array}{l}\text { Hydrate composition } \\
(\exp )\end{array}$} & \multicolumn{2}{|c|}{$\begin{array}{l}\text { Hydrate composition } \\
\text { (pre) }\end{array}$} \\
\hline & & & & $\mathrm{CO}_{2}$ & $\mathrm{CH}_{4}$ & $\mathrm{CO}_{2}$ & $\mathrm{CH}_{4}$ & $\mathrm{CO}_{2}$ & $\mathrm{CH}_{4}$ \\
\hline 273.1 & 2.00 & 1.92 & I & 0.28 & 0.72 & 0.92 & 0.09 & 0.45 & 0.55 \\
\hline 274.1 & 2.00 & 2.03 & I & 0.40 & 0.60 & 0.94 & 0.06 & 0.58 & 0.42 \\
\hline 275.4 & 2.00 & 2.06 & I & 0.61 & 0.39 & 0.98 & 0.02 & 0.75 & 0.25 \\
\hline 276.3 & 2.00 & 2.04 & I & 0.79 & 0.21 & 1.00 & 0.00 & 0.88 & 0.12 \\
\hline 273.8 & 2.60 & 2.48 & I & 0.13 & 0.87 & 0.66 & 0.34 & 0.24 & 0.76 \\
\hline 274.9 & 2.60 & 2.54 & I & 0.23 & 0.77 & 0.85 & 0.15 & 0.38 & 0.62 \\
\hline 276.3 & 2.60 & 2.56 & I & 0.42 & 0.59 & 0.93 & 0.07 & 0.58 & 0.42 \\
\hline 277.5 & 2.60 & 2.55 & I & 0.64 & 0.36 & 0.98 & 0.02 & 0.77 & 0.23 \\
\hline 278.1 & 2.60 & 2.46 & I & 0.83 & 0.17 & 0.99 & 0.01 & 0.90 & 0.10 \\
\hline 276.6 & 3.50 & 3.29 & I & 0.13 & 0.87 & 0.65 & 0.35 & 0.24 & 0.76 \\
\hline 277.6 & 3.50 & 3.32 & I & 0.25 & 0.75 & 0.73 & 0.27 & 0.40 & 0.60 \\
\hline 279.0 & 3.50 & 3.45 & I & 0.42 & 0.58 & 0.89 & 0.11 & 0.57 & 0.43 \\
\hline 279.9 & 3.50 & 3.43 & I & 0.61 & 0.39 & 0.95 & 0.05 & 0.74 & 0.26 \\
\hline 280.5 & 3.50 & 3.30 & I & 0.83 & 0.17 & 0.99 & 0.01 & 0.90 & 0.10 \\
\hline \multicolumn{2}{|c|}{ AAD } & $3.3 \%$ & & & & & & \multicolumn{2}{|c|}{0.29} \\
\hline
\end{tabular}

323 As it is clear in Table 10, Table 11 and Figure 7, the thermodynamic model predicts the 324 equilibrium pressure with adequate deviations (average deviations for methane-carbon dioxide 325 and nitrogen-carbon dioxide hydrate pressure were 3.3 and 5.7\%, respectively).

326 As seen in Table 10, at low concentrations of nitrogen, the hydrate phase compositions were 327 acceptably simulated (sI). Nevertheless, when nitrogen was the dominate component in hydrate 
phase, the simulation results deviated from the experimental data. Hence, the hydrate phase compositions were again simulated by taking into account both structures, sI and sII. Prediction results for two isotherms of $\mathrm{N}_{2} / \mathrm{CO}_{2}$ binary hydrate are presented in Figure 8. This figure shows that, at higher pressures, the hydrate preferentially encapsulates nitrogen from the $\mathrm{N}_{2} / \mathrm{CO}_{2}$ mixture. Consequently, the nitrogen composition in hydrate phase increases. As clear on the figure, at high concentrations of nitrogen in hydrate phase, sII simulation results are in better agreement with experimental data compared to sI results. This can be explained by the fact that pure nitrogen forms sII hydrates. Thus, when nitrogen is the major component in the mixture, there might be a structural transition from sI to sII.

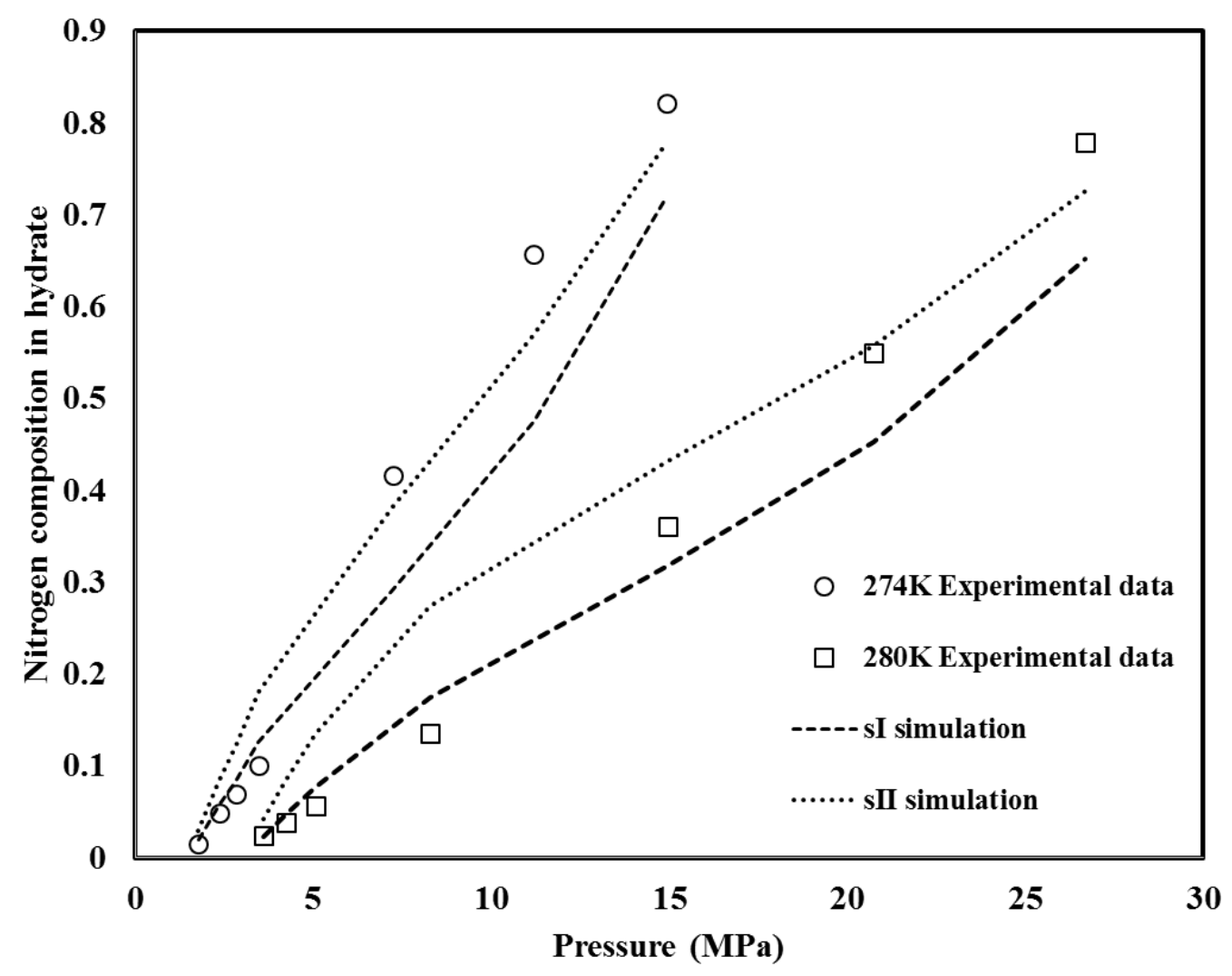

Figure 8. P-x diagram of $\mathrm{N}_{2}-\mathrm{CO}_{2}$ hydrate based on the experimental results of Seo et al. (sI) [70] and our simulation results

For the methane-carbon dioxide mixture, although the equilibrium pressure were satisfactorily simulated (average absolute deviation 3.3\%), the thermodynamic model failed to evaluate the hydrate composition (average absolute deviation 0.29 mole fraction).

\subsection{Using a tracer}

Using a tracer in gas or liquid phase could help to measure hydrate composition. A tracer must not be able to form hydrates and only a small amount should be utilized. This section presents a short report of publications which have used a tracer in their experiment to measure hydrate 
composition. Of course, based on the experimental results of these papers, the capability of the thermodynamic model to simulate hydrate composition is evaluated.

$349 \mathrm{Ng}$ determined the hydrate composition for six gas mixture including methane, ethane, propane, 350 iso-butane, n-butane and carbon dioxide. He used n-pentane as a tracer in gas phase. Thanks to a 351 gasometer and composition of n-pentane at the end of experiments, he successfully determined 352 the amount of original gas mixture and overall gas dissociation [71]. Table 12 shows the different 353 gas mixtures in the study. Ng's experimental data and our simulation results are presented in 354 Table 13.

Table 12. Feed composition of different mixtures [71]

\begin{tabular}{|l|c|c|c|c|c|c|}
\hline \multirow{2}{*}{\multicolumn{1}{|c|}{ Gas }} & \multicolumn{5}{|c|}{ Mixtures (Concentration mole \%) $( \pm 0.003)$} \\
\cline { 2 - 7 } & $\mathrm{a}$ & $\mathrm{b}$ & $\mathrm{c}$ & $\mathrm{d}$ & $\mathrm{e}$ & $\mathrm{f}$ \\
\hline Carbon dioxide & 0 & 0 & 0 & 2 & 0 & 2 \\
\hline Methane & 90 & 99 & 84 & 88 & 80 & 86.5 \\
\hline Ethane & 0 & 0 & 10 & 0 & 10 & 0 \\
\hline Propane & 10 & 1 & 4 & 8 & 5 & 4 \\
\hline iso-Butane & 0 & 0 & 2 & 2 & 2 & 0.5 \\
\hline n-Butane & 0 & 0 & 0 & 0 & 3 & 1 \\
\hline
\end{tabular}

Table 13. Experimental results of $\mathrm{Ng}$ [71] and our modeling results

\begin{tabular}{|c|c|c|c|c|c|c|c|c|c|c|c|c|c|c|}
\hline \multirow{2}{*}{$\begin{array}{c}\mathrm{T}(\mathrm{C}) \\
( \pm 0.05)\end{array}$} & \multirow{2}{*}{$\begin{array}{c}\mathrm{P} \\
(\mathrm{MPa}) \\
( \pm 0.007)\end{array}$} & \multirow{2}{*}{ Feed } & \multicolumn{6}{|c|}{ Experimental hydrate composition $( \pm 0.003)$} & \multicolumn{6}{|c|}{ Hydrate composition simulation } \\
\hline & & & $\mathrm{C}_{1}$ & $\mathrm{C}_{2}$ & $\mathrm{C}_{3}$ & $\mathrm{iC}_{4}$ & $\mathrm{nC}_{4}$ & $\mathrm{CO}_{2}$ & $\mathrm{C}_{1}$ & $\mathrm{C}_{2}$ & $\mathrm{C}_{3}$ & $\mathrm{iC}_{4}$ & $\mathrm{nC}_{4}$ & $\mathrm{CO}_{2}$ \\
\hline 11.4 & 2.07 & $\mathrm{a}$ & 0.586 & - & 0.414 & - & - & - & 0.61 & - & 0.39 & - & - & - \\
\hline 20.1 & 6.89 & $\mathrm{a}$ & 0.657 & - & 0.343 & - & - & - & 0.64 & - & 0.36 & - & - & - \\
\hline 3.7 & 2.07 & $\mathrm{~b}$ & 0.726 & - & 0.274 & - & - & - & 0.65 & - & 0.35 & - & - & - \\
\hline 13.2 & 6.89 & $\mathrm{~b}$ & 0.771 & - & 0.229 & - & - & - & 0.67 & - & 0.33 & - & - & - \\
\hline \multicolumn{9}{|c|}{ AADc } & \multicolumn{6}{|c|}{0.05} \\
\hline 10.9 & 2.07 & $\mathrm{c}$ & 0.606 & 0.087 & 0.182 & 0.124 & - & - & 0.62 & 0.01 & 0.2 & 0.17 & - & - \\
\hline 19.4 & 6.89 & $\mathrm{c}$ & 0.59 & 0.087 & 0.2 & 0.123 & - & - & 0.64 & 0.01 & 0.2 & 0.15 & - & - \\
\hline \multicolumn{9}{|c|}{ AADc } & 0.03 & 0.07 & 0.009 & 0.02 & & \\
\hline 12.9 & 2.07 & $\mathrm{~d}$ & 0.6 & - & 0.297 & 0.092 & - & 0.0102 & 0.6 & - & 0.28 & 0.1 & - & 0.02 \\
\hline 21.6 & 6.89 & $\mathrm{~d}$ & 0.63 & - & 0.289 & 0.078 & - & 0.0104 & 0.62 & - & 0.27 & 0.09 & - & 0.02 \\
\hline \multicolumn{9}{|c|}{ AADc } & 0.005 & - & 0.018 & 0.01 & - & 0.01 \\
\hline 11.3 & 2.07 & $\mathrm{e}$ & 0.581 & 0.074 & 0.206 & 0.107 & 0.031 & - & 0.62 & 0.01 & 0.22 & 0.14 & 0.01 & - \\
\hline 19.6 & 6.89 & $\mathrm{e}$ & 0.617 & 0.089 & 0.181 & 0.087 & 0.027 & - & 0.64 & 0.01 & 0.23 & 0.11 & 0.01 & - \\
\hline \multicolumn{9}{|c|}{ AADc } & 0.031 & 0.07 & 0.03 & 0.02 & 0.02 & \\
\hline 9.8 & 2.07 & $\mathrm{f}$ & 0.584 & 0.076 & 0.265 & 0.044 & 0.018 & 0.011 & 0.6 & 0.006 & 0.31 & 0.06 & 0.007 & 0.017 \\
\hline 18.7 & 6.89 & $\mathrm{f}$ & 0.621 & 0.077 & 0.238 & 0.039 & 0.011 & 0.01 & 0.63 & 0.007 & 0.29 & 0.05 & 0.006 & 0.017 \\
\hline \multicolumn{9}{|c|}{ AADc } & 0.01 & 0.07 & 0.05 & 0.01 & 0.008 & 0.006 \\
\hline
\end{tabular}

358

359 Obviously, in Table 13, there is a wide range of average absolute deviation according to the 360 number and type of components in the feed gas, between 0.006 and 0.07 . This variation could be 
explained by the fact that for the ternary or quaternary mixtures, kinetic considerations might affect experimental results. Note: the largest deviation belongs to ethane in all the mixtures.

In the 21st century, researchers at Ecole National Superieur des Mines de Saint-Etienne (Mines Saint-Etienne) developed a new method to determine the hydrate composition not only at equilibrium condition, but also during the crystallization under non-equilibrium conditions. Their new procedure was based on tracing the concentration of lithium salt in liquid phase and material balance. The protocol is as follows: after gas injection inside the reactor, water solution with 10 ppm concentration of lithium salt was inserted in the reactor. By decreasing the temperature at a desire value, hydrate formation started. After several days, the system reached at equilibrium. At this point a gas and liquid sample was taken. The gas sample was analyzed by gas chromatography to determine the gas composition in vapor phase. The liquid sample was analyzed by ion chromatograph to measure the concentration of lithium salt in liquid phase. As lithium does not participate in hydrate formation, it is possible to calculate the amount of water at equilibrium according to the lithium concentration. Then, the system temperature increased stepwise and gas and liquid samples were taken [14,57]. A diagram of their experiments is shown in Figure 9.

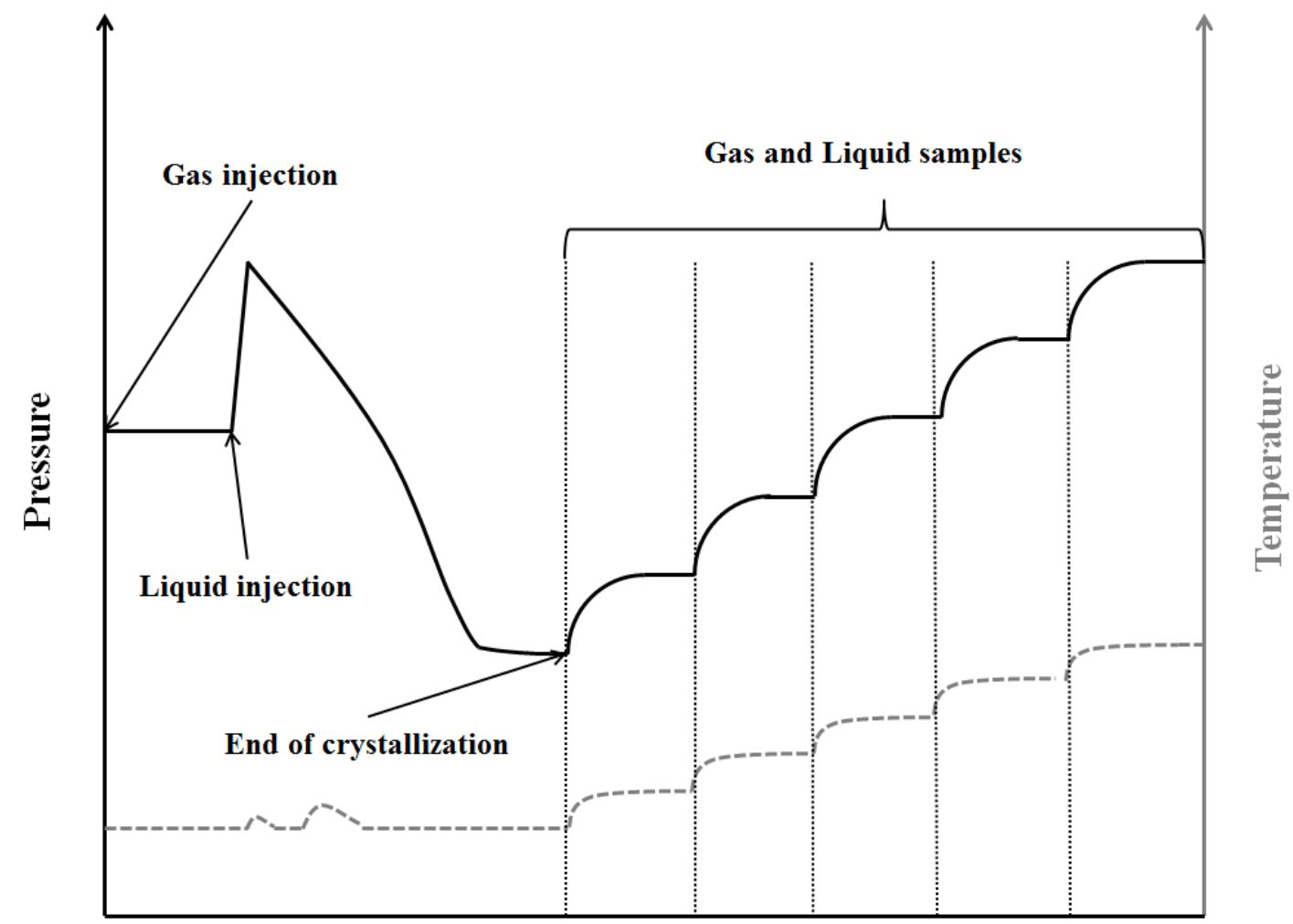

Time

Figure 9. The diagram of experimental procedure

Based on this procedure, Herri et al. [14] studied the equilibrium conditions of carbon dioxidenitrogen and carbon dioxide-methane hydrates. Table 14 and Table 15 present their experimental 
381 results and our corresponding simulation results for carbon dioxide-nitrogen and carbon dioxidemethane mixtures, respectively.

Table 14. Experimental results from Herri et al. [14] and simulation results for carbon dioxide-nitrogen mixture

\begin{tabular}{|c|c|c|c|c|c|c|c|c|c|}
\hline \multirow{2}{*}{$\begin{array}{l}\mathrm{T}(\mathrm{K}) \\
( \pm 0.1)\end{array}$} & \multirow{2}{*}{$\begin{array}{c}\text { Pexp } \\
\text { (MPa) } \\
( \pm 0.01)\end{array}$} & \multirow{2}{*}{$\begin{array}{l}\text { Ppre } \\
(\mathrm{MPa})\end{array}$} & \multirow{2}{*}{$S$} & \multicolumn{2}{|c|}{$\begin{array}{c}\text { Gas composition (exp) } \\
( \pm 0.001)\end{array}$} & \multicolumn{2}{|c|}{$\begin{array}{l}\text { Hydrate composition } \\
(\exp )( \pm 0.003)\end{array}$} & \multicolumn{2}{|c|}{$\begin{array}{l}\text { Hydrate composition } \\
\text { (pre) }\end{array}$} \\
\hline & & & & $\mathrm{CO}_{2}$ & $\mathrm{~N}_{2}$ & $\mathrm{CO}_{2}$ & $\mathrm{~N}_{2}$ & $\mathrm{CO}_{2}$ & $\mathrm{~N}_{2}$ \\
\hline 273.4 & 6.10 & 7.51 & I & 0.16 & 0.84 & 0.66 & 0.34 & 0.63 & 0.37 \\
\hline 274.5 & 6.20 & 8.31 & I & 0.16 & 0.84 & 0.66 & 0.34 & 0.63 & 0.37 \\
\hline 275.4 & 6.40 & 8.64 & I & 0.19 & 0.82 & 0.66 & 0.34 & 0.66 & 0.34 \\
\hline 276.5 & 6.60 & 9.42 & I & 0.20 & 0.80 & 0.58 & 0.42 & 0.67 & 0.33 \\
\hline 273.9 & 5.90 & 5.40 & I & 0.25 & 0.75 & 0.75 & 0.25 & 0.77 & 0.23 \\
\hline 274.7 & 5.90 & 5.93 & I & 0.26 & 0.75 & 0.73 & 0.27 & 0.76 & 0.24 \\
\hline 276.0 & 5.90 & 6.90 & I & 0.26 & 0.74 & 0.70 & 0.30 & 0.76 & 0.24 \\
\hline 276.9 & 6.00 & 7.70 & I & 0.27 & 0.74 & 0.70 & 0.30 & 0.75 & 0.25 \\
\hline 277.8 & 6.30 & 8.14 & I & 0.29 & 0.71 & 0.67 & 0.33 & 0.77 & 0.23 \\
\hline 278.1 & 6.40 & 8.41 & I & 0.30 & 0.71 & 0.69 & 0.31 & 0.77 & 0.23 \\
\hline 278.4 & 6.40 & 8.82 & I & 0.30 & 0.71 & 0.72 & 0.29 & 0.76 & 0.24 \\
\hline 278.6 & 6.50 & 8.93 & I & 0.30 & 0.70 & 0.70 & 0.31 & 0.77 & 0.23 \\
\hline 275.4 & 6.10 & 7.99 & I & 0.20 & 0.80 & 0.67 & 0.33 & 0.69 & 0.31 \\
\hline 276.0 & 6.20 & 8.14 & I & 0.22 & 0.78 & 0.65 & 0.35 & 0.70 & 0.30 \\
\hline 280.1 & 5.30 & 5.75 & I & 0.56 & 0.44 & 0.85 & 0.16 & 0.91 & 0.09 \\
\hline 281.1 & 5.60 & 6.42 & I & 0.59 & 0.42 & 0.82 & 0.18 & 0.91 & 0.09 \\
\hline \multicolumn{2}{|c|}{$\mathrm{AADp}$} & $25.6 \%$ & \multicolumn{5}{|c|}{ AADc } & \multicolumn{2}{|c|}{0.05} \\
\hline
\end{tabular}

385

\begin{tabular}{|c|c|c|c|c|c|c|c|c|c|}
\hline \multirow{2}{*}{$\begin{array}{c}\mathrm{T}(\mathrm{K}) \\
( \pm 0.1)\end{array}$} & $\begin{array}{c}\text { Pexp } \\
(\mathrm{MPa}) \\
( \pm 0.01)\end{array}$ & \multirow{2}{*}{$\begin{array}{c}\text { Ppre } \\
(\mathrm{MPa})\end{array}$} & \multirow{2}{*}{$\mathrm{S}$} & $\begin{array}{c}\text { Gas composition (exp) } \\
( \pm 0.001)\end{array}$ & $\begin{array}{c}\text { Hydrate composition } \\
(\exp )( \pm 0.003)\end{array}$ & \multicolumn{2}{|c|}{$\begin{array}{c}\text { Hydrate composition } \\
(\mathrm{pre})\end{array}$} \\
\cline { 5 - 11 } & & & $\mathrm{CO}_{2}$ & $\mathrm{CH}_{4}$ & $\mathrm{CO}_{2}$ & $\mathrm{CH}_{4}$ & $\mathrm{CO}_{2}$ & $\mathrm{CH}_{4}$ \\
\hline 277.15 & 2.04 & 2.03 & $\mathrm{I}$ & 1.00 & 0.00 & 1.00 & 0.00 & 1.00 & 0.00 \\
\hline 277.15 & 2.36 & 2.45 & $\mathrm{I}$ & 0.64 & 0.36 & 0.77 & 0.23 & 0.77 & 0.23 \\
\hline 277.15 & 2.55 & 2.63 & $\mathrm{I}$ & 0.52 & 0.48 & 0.68 & 0.32 & 0.68 & 0.32 \\
\hline 277.15 & 2.80 & 2.92 & $\mathrm{I}$ & 0.36 & 0.64 & 0.54 & 0.47 & 0.53 & 0.47 \\
\hline 277.15 & 3.55 & 3.55 & $\mathrm{I}$ & 0.11 & 0.89 & 0.21 & 0.79 & 0.20 & 0.80 \\
\hline 277.15 & 3.90 & 3.94 & $\mathrm{I}$ & 0.00 & 1.00 & 0.00 & 1.00 & 0.00 & 1.00 \\
\hline \multicolumn{2}{|c|}{ AADp } & $2.10 \%$ & \multicolumn{6}{|c|}{ AADc } & \multicolumn{2}{|c|}{0.004} \\
\hline
\end{tabular}

388

389 In the case of carbon dioxide-nitrogen, the simulation results for hydrate equilibrium pressure are 390 not very reliable. Nonetheless, the average absolute deviation for prediction of hydrate 
composition is small (0.05). For carbon dioxide-methane mixture, the simulation results are in respectable agreement with the experimental results. As it can be seen in Table 15, the average deviation of equilibrium pressure was about $2 \%$. The thermodynamic model better predicts the hydrate composition. The average deviation for carbon dioxide and methane compositions in hydrate phase was 0.004. Figure 10 presents clearly the excellent match between the experimental and simulation results for carbon dioxide-methane hydrate mixture.

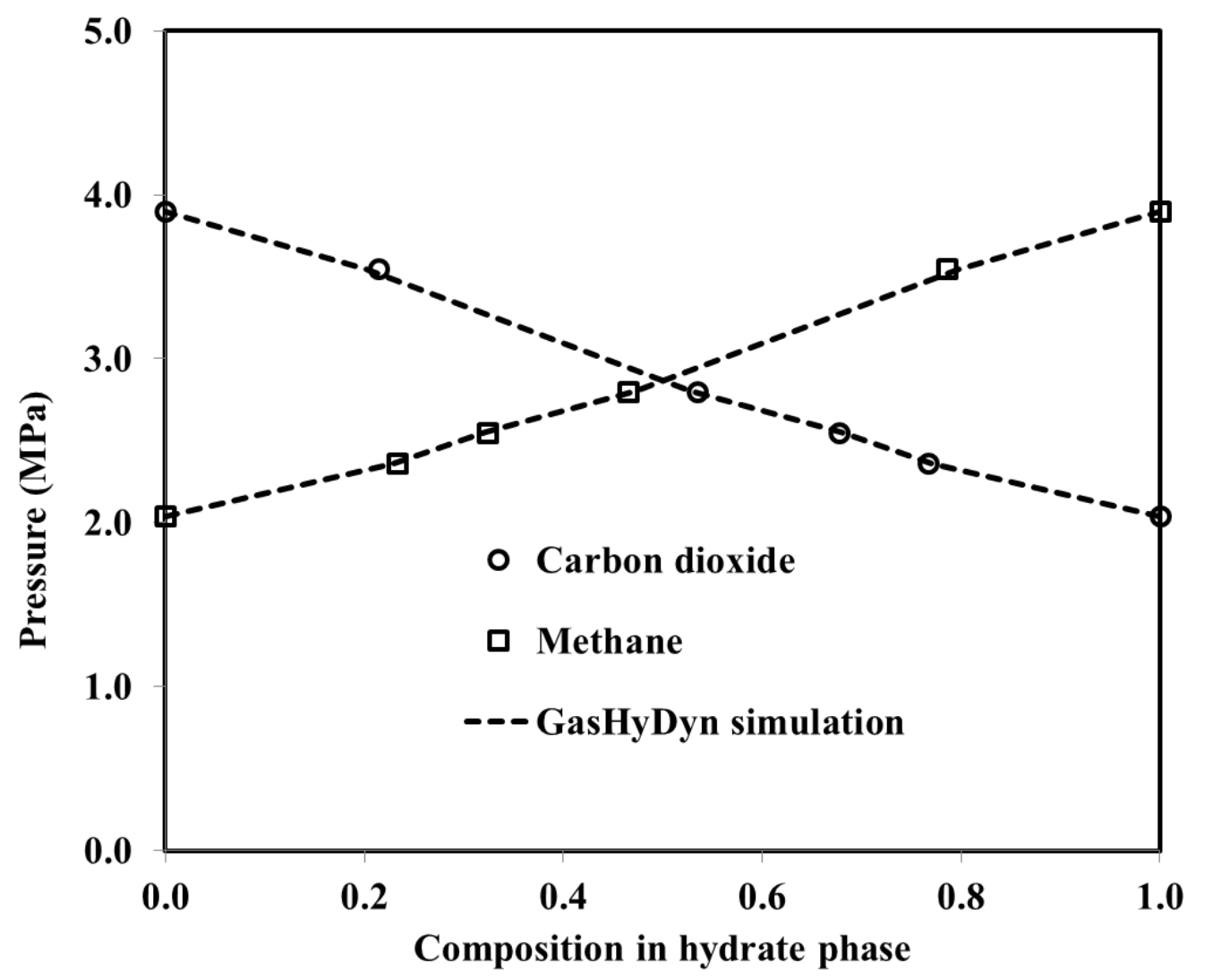

Figure 10. Experimental data from Herri et al. [14] for methane-carbon dioxide hydrate (sI) and simulation results

400 Le Quang et al. studied the equilibrium conditions of several gas mixtures from $\mathrm{N}_{2}-\mathrm{CO}_{2}-\mathrm{CH}_{4}-$ $401 \mathrm{C}_{2} \mathrm{H}_{6}-\mathrm{C}_{3} \mathrm{H}_{8}-\mathrm{nC}_{4} \mathrm{H}_{10}$. They also determined the guest composition in vapor and hydrate phases 402 [57]. They used the same method as Herri et al [14] to analyze guest composition in different 403 phases. In addition, they studied the influence of crystallization rate on the final equilibrium 404 pressure and composition during the crystallization of $\mathrm{CO}_{2}-\mathrm{CH}_{4}-\mathrm{C}_{2} \mathrm{H}_{6}$ mixed hydrate. All the 405 experimental data and our equivalent simulation results are listed in Table 16, Table 17 and Table 18. They reported that, for the equilibrium points near total dissociation, hydrate composition was suspected to have large errors. Thus, they were not used for our purposes. As Table 16 shows, the 409 thermodynamic model realistically predicts the equilibrium pressures of binary mixtures including carbon dioxide. The average deviation of the equilibrium pressure predictions for $\mathrm{CO}_{2}$ - 
$410 \mathrm{CH}_{4}$ and $\mathrm{CO}_{2}-\mathrm{N}_{2}$ are about 2 and 3\%, respectively. For hydrate composition extrapolation, the 411 average absolute deviations are 0.05 and 0.04 for $\mathrm{CO}_{2}-\mathrm{CH}_{4}$ and $\mathrm{CO}_{2}-\mathrm{N}_{2}$, respectively.

Table 16. Carbon dioxide-methane and carbon dioxide-nitrogen hydrate mixtures, experimental data [57] and simulation results

\begin{tabular}{|c|c|c|c|c|c|c|c|c|c|c|c|c|c|}
\hline \multirow[t]{2}{*}{ Gas } & \multirow{2}{*}{$\begin{array}{c}\text { Texp } \\
\left({ }^{\circ} \mathrm{C}\right) \\
( \pm 0.1)\end{array}$} & \multirow{2}{*}{$\begin{array}{l}\text { Pexp } \\
\text { (MPa) } \\
( \pm 0.01)\end{array}$} & \multirow{2}{*}{$\begin{array}{l}\text { Ppre } \\
\text { (MPa) }\end{array}$} & \multirow[t]{2}{*}{$\mathrm{S}$} & \multicolumn{3}{|c|}{$\begin{array}{l}\text { Gas composition } \\
(\exp )( \pm 0.001)\end{array}$} & \multicolumn{3}{|c|}{$\begin{array}{l}\text { Hydrate composition } \\
(\exp )( \pm 0.003)\end{array}$} & \multicolumn{3}{|c|}{ Hydrate composition (Pre) } \\
\hline & & & & & $\mathrm{N}_{2}$ & $\mathrm{CO}_{2}$ & $\mathrm{CH}_{4}$ & $\mathrm{~N}_{2}$ & $\mathrm{CO}_{2}$ & $\mathrm{CH}_{4}$ & $\mathrm{~N}_{2}$ & $\mathrm{CO}_{2}$ & $\mathrm{CH}_{4}$ \\
\hline \multirow{12}{*}{$\begin{array}{l}\underbrace{+} \\
\text { İ } \\
\overbrace{}^{\prime}\end{array}$} & 3.4 & 3.33 & 3.29 & I & - & 0.13 & 0.87 & - & 0.32 & 0.68 & - & 0.23 & 0.77 \\
\hline & 4.4 & 3.53 & 3.62 & $\mathrm{I}$ & - & 0.13 & 0.87 & - & 0.32 & 0.68 & - & 0.24 & 0.76 \\
\hline & 4.9 & 3.71 & 3.80 & I & - & 0.14 & 0.86 & - & 0.32 & 0.68 & - & 0.24 & 0.76 \\
\hline & 5.8 & 4.03 & 4.14 & I & - & 0.15 & 0.85 & - & 0.32 & 0.68 & - & 0.26 & 0.74 \\
\hline & 6.8 & 4.45 & 4.57 & I & - & 0.16 & 0.84 & - & 0.32 & 0.68 & - & 0.27 & 0.73 \\
\hline & 7.8 & 4.93 & 5.06 & I & - & 0.18 & 0.83 & - & 0.30 & 0.70 & - & 0.28 & 0.72 \\
\hline & 2.2 & 2.91 & 2.93 & I & - & 0.12 & 0.88 & - & 0.29 & 0.71 & - & 0.22 & 0.78 \\
\hline & 2.5 & 2.97 & 3.00 & I & - & 0.13 & 0.87 & - & 0.28 & 0.72 & - & 0.23 & 0.77 \\
\hline & 3.6 & 3.18 & 3.33 & I & - & 0.14 & 0.87 & - & 0.28 & 0.72 & - & 0.24 & 0.76 \\
\hline & 4.5 & 3.47 & 3.63 & I & - & 0.15 & 0.85 & - & 0.27 & 0.73 & - & 0.25 & 0.75 \\
\hline & 5.2 & 3.80 & 3.85 & I & - & 0.16 & 0.84 & - & 0.23 & 0.77 & - & 0.27 & 0.73 \\
\hline & \multicolumn{2}{|c|}{$\mathrm{AADp}$} & $2.4 \%$ & \multicolumn{7}{|c|}{ AADc } & \multicolumn{3}{|c|}{0.05} \\
\hline \multirow{6}{*}{ o } & 2.3 & 2.46 & 2.53 & I & 0.33 & 0.67 & - & 0.06 & 0.94 & - & 0.05 & 0.95 & - \\
\hline & 3.1 & 2.60 & 2.69 & I & 0.31 & 0.69 & - & 0.07 & 0.93 & - & 0.04 & 0.96 & - \\
\hline & 3.3 & 2.66 & 2.73 & I & 0.30 & 0.70 & - & 0.07 & 0.93 & - & 0.04 & 0.96 & - \\
\hline & 4.3 & 2.87 & 2.99 & I & 0.28 & 0.72 & - & 0.09 & 0.91 & - & 0.04 & 0.96 & - \\
\hline & 5.2 & 3.13 & 3.22 & I & 0.25 & 0.75 & - & 0.12 & 0.88 & - & 0.03 & 0.97 & - \\
\hline & \multicolumn{2}{|c|}{$\mathrm{AADp}$} & $3.16 \%$ & \multicolumn{7}{|c|}{ AADc } & \multicolumn{3}{|c|}{0.04} \\
\hline
\end{tabular}




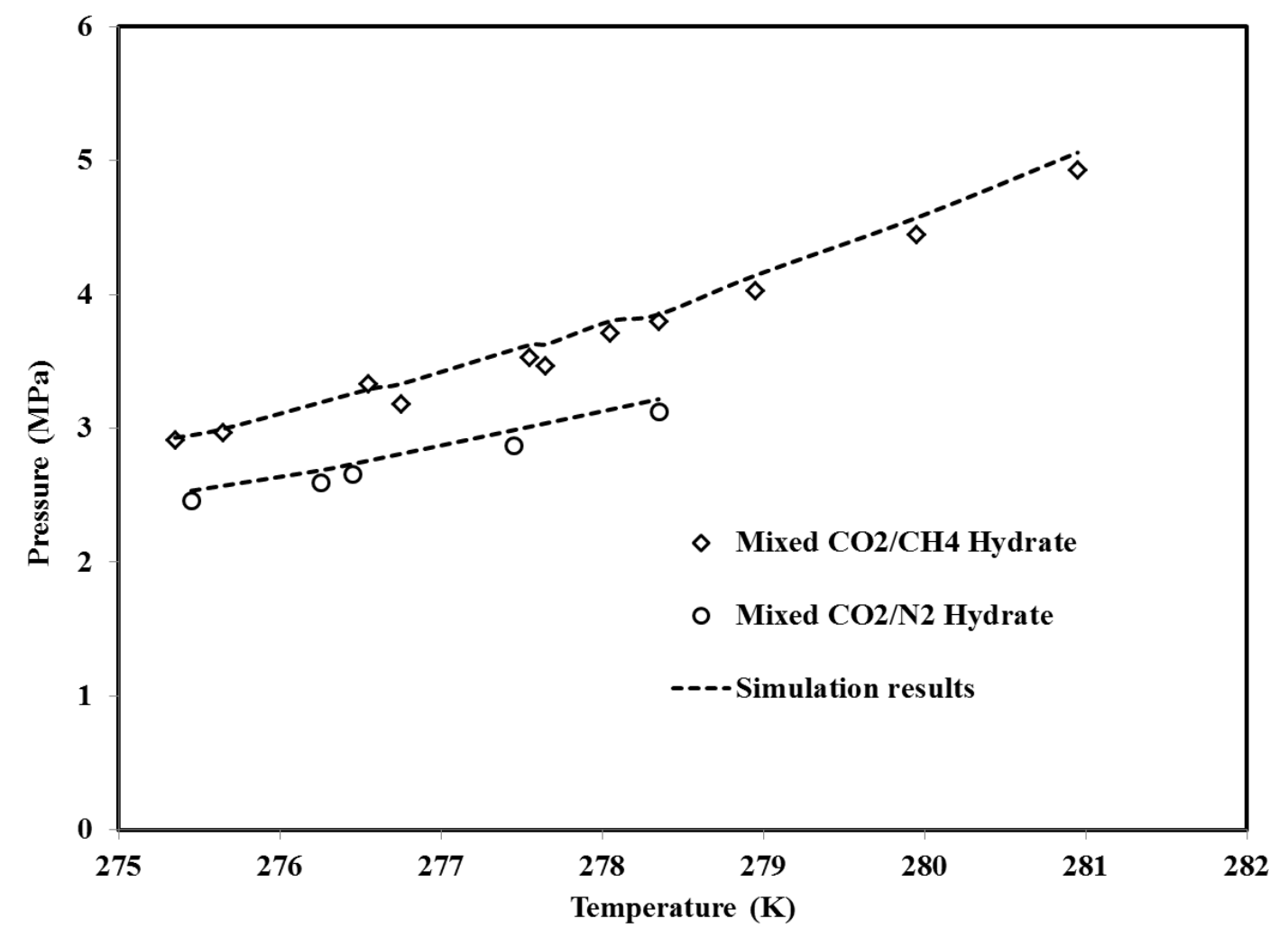

Figure 11. P-T diagram of mixed $\mathrm{CO}_{2} / \mathrm{CH}_{4}$ and $\mathrm{CO}_{2} / \mathrm{N}_{2}$ hydrates, experimental data [57] and simulation results (sI). The vapor and hydrate compositions were listed in Table 16.

419 As aforementioned, Le Quang et al. investigated the effect of crystallization rate on hydrate hydrate equilibrium pressure and composition at final state differed according to the rate of crystallization. They concluded that at quick crystallization rate, mixed gas hydrate could not be at thermodynamic equilibrium [57]. Table 17 reveals that the results of thermodynamic model is closer to the slow crystallization data. The average deviations of equilibrium pressure prediction

425 for slow and quick crystallization are about 2 and 8\%, respectively. The average deviations for carbon dioxide in hydrate phase for slow and quick crystallization are similar (0.01). This is not the case for methane and ethane compositions in hydrate phase, where the deviation of simulation for slow crystallization are much better compared to quick rate (e.g. ethane, 0.009 for slow compared to 0.05 for quick). 
Table 17. Experimental results of Carbon dioxide-methane-ethane hydrate from Le Quang et al. [57] and simulation results

\begin{tabular}{|c|c|c|c|c|c|c|c|c|c|c|c|c|c|}
\hline \multirow[t]{2}{*}{ Gas } & \multirow{2}{*}{$\begin{array}{c}\text { Texp } \\
\left({ }^{\circ} \mathrm{C}\right) \\
( \pm 0.1) \\
\end{array}$} & \multirow{2}{*}{$\begin{array}{c}\text { Pexp } \\
(\mathrm{MPa}) \\
( \pm 0.01) \\
\end{array}$} & \multirow{2}{*}{$\begin{array}{l}\text { Ppre } \\
\text { (MPa) }\end{array}$} & \multirow[t]{2}{*}{$S$} & \multicolumn{3}{|c|}{$\begin{array}{l}\text { Gas composition } \\
(\exp )( \pm 0.001)\end{array}$} & \multicolumn{3}{|c|}{$\begin{array}{l}\text { Hydrate composition } \\
\quad(\exp )( \pm 0.003)\end{array}$} & \multicolumn{3}{|c|}{$\begin{array}{l}\text { Hydrate composition } \\
\text { (Pre) }\end{array}$} \\
\hline & & & & & $\mathrm{CO}_{2}$ & $\mathrm{CH}_{4}$ & $\mathrm{C}_{2} \mathrm{H}_{6}$ & $\mathrm{CO}_{2}$ & $\mathrm{CH}_{4}$ & $\mathrm{C}_{2} \mathrm{H}_{6}$ & $\mathrm{CO}_{2}$ & $\mathrm{CH}_{4}$ & $\mathrm{C}_{2} \mathrm{H}_{6}$ \\
\hline \multirow{7}{*}{ 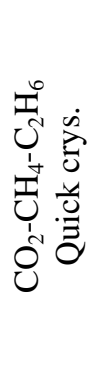 } & 2.75 & 3.54 & 3.11 & I & 0.03 & 0.96 & 0.01 & 0.07 & 0.89 & 0.04 & 0.05 & 0.87 & 0.08 \\
\hline & 3.65 & 3.81 & 3.35 & $\mathrm{I}$ & 0.03 & 0.96 & 0.01 & 0.07 & 0.89 & 0.04 & 0.05 & 0.86 & 0.09 \\
\hline & 5.15 & 4.23 & 3.79 & I & 0.03 & 0.95 & 0.02 & 0.07 & 0.89 & 0.04 & 0.05 & 0.83 & 0.11 \\
\hline & 6.55 & 4.56 & 4.33 & I & 0.03 & 0.95 & 0.02 & 0.07 & 0.89 & 0.04 & 0.06 & 0.83 & 0.12 \\
\hline & 7.80 & 5.12 & 4.76 & I & 0.04 & 0.94 & 0.02 & 0.07 & 0.89 & 0.04 & 0.06 & 0.81 & 0.13 \\
\hline & 9.25 & 5.99 & 5.67 & $\mathrm{I}$ & 0.04 & 0.94 & 0.02 & 0.04 & 0.90 & 0.07 & 0.07 & 0.82 & 0.12 \\
\hline & \multicolumn{2}{|c|}{$\mathrm{AADp}$} & $8.6 \%$ & \multicolumn{7}{|c|}{ AADc } & 0.01 & 0.05 & 0.05 \\
\hline \multirow{6}{*}{ 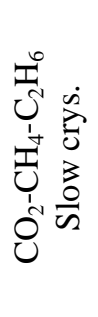 } & 4.60 & 3.78 & 3.64 & I & 0.04 & 0.95 & 0.01 & 0.08 & 0.80 & 0.12 & 0.06 & 0.84 & 0.09 \\
\hline & 4.20 & 3.56 & 3.50 & $\mathrm{I}$ & 0.03 & 0.95 & 0.01 & 0.08 & 0.83 & 0.09 & 0.06 & 0.84 & 0.10 \\
\hline & 3.25 & 3.18 & 3.27 & $\mathrm{I}$ & 0.03 & 0.96 & 0.01 & 0.08 & 0.85 & 0.08 & 0.06 & 0.87 & 0.07 \\
\hline & 1.30 & 2.76 & 2.76 & $\mathrm{I}$ & 0.03 & 0.96 & 0.01 & 0.08 & 0.86 & 0.06 & 0.06 & 0.89 & 0.05 \\
\hline & 4.20 & 3.57 & 3.46 & I & 0.04 & 0.95 & 0.02 & 0.06 & 0.84 & 0.09 & 0.07 & 0.83 & 0.10 \\
\hline & \multicolumn{2}{|c|}{ AADp } & $2.2 \%$ & \multicolumn{7}{|c|}{$\mathrm{AADc}$} & 0.01 & 0.02 & 0.01 \\
\hline
\end{tabular}

435

436 As seen in Table 18, the average deviation of the equilibrium pressures calculated for the 437 quaternary mixture of methane-ethane-propane-butane is about $13 \%$ which is expected due to 438 more significant kinetic effects in quaternary mixtures.

439 Hydrate composition for the methane and butane is well simulated (AADc=0.01), while for 440 ethane and propane, the average deviation of hydrate composition are 0.06 and 0.07 , respectively.

Table 18. Experimental data of methane-ethane-propane-butane hydrate from Le Quang et al. [57] and simulation results

\begin{tabular}{|c|c|c|c|c|c|c|c|c|c|c|c|c|c|c|c|}
\hline \multirow{2}{*}{$\begin{array}{l}\text { Texp } \\
\left({ }^{\circ} \mathrm{C}\right) \\
( \pm 0.1)\end{array}$} & \multirow{2}{*}{$\begin{array}{c}\text { Pexp } \\
\text { (MPa) } \\
( \pm 0.01)\end{array}$} & \multirow{2}{*}{$\begin{array}{l}\text { Ppre } \\
(\mathrm{MPa})\end{array}$} & \multirow[t]{2}{*}{$S$} & \multicolumn{4}{|c|}{$\begin{array}{l}\text { Gas composition (exp) } \\
( \pm 0.001)\end{array}$} & \multicolumn{4}{|c|}{$\begin{array}{l}\text { Hydrate composition (exp) } \\
\qquad( \pm 0.003)\end{array}$} & \multicolumn{4}{|c|}{ Hydrate composition (Pre) } \\
\hline & & & & $\mathrm{C}_{1}$ & $\mathrm{C}_{2}$ & $\mathrm{C}_{3}$ & $\mathrm{C}_{4}$ & $\mathrm{C}_{1}$ & $\mathrm{C}_{2}$ & $\mathrm{C}_{3}$ & $\mathrm{C}_{4}$ & $\mathrm{C}_{1}$ & $\mathrm{C}_{2}$ & $\mathrm{C}_{3}$ & $\mathrm{C}_{4}$ \\
\hline 2.40 & 2.28 & 1.70 & II & 0.97 & 0.02 & 0.00 & 0.01 & 0.73 & 0.11 & 0.11 & 0.05 & 0.75 & 0.03 & 0.16 & 0.06 \\
\hline 3.45 & 2.31 & 1.82 & II & 0.97 & 0.02 & 0.01 & 0.01 & 0.73 & 0.11 & 0.11 & 0.05 & 0.74 & 0.03 & 0.18 & 0.06 \\
\hline 7.60 & 2.75 & 2.50 & II & 0.94 & 0.04 & 0.01 & 0.02 & 0.71 & 0.10 & 0.14 & 0.05 & 0.72 & 0.04 & 0.18 & 0.07 \\
\hline 9.15 & 2.97 & 2.70 & II & 0.93 & 0.04 & 0.01 & 0.02 & 0.68 & 0.10 & 0.17 & 0.05 & 0.70 & 0.03 & 0.20 & 0.06 \\
\hline 9.90 & 3.05 & 2.78 & II & 0.92 & 0.04 & 0.02 & 0.02 & 0.68 & 0.10 & 0.17 & 0.05 & 0.70 & 0.03 & 0.22 & 0.05 \\
\hline 10.80 & 3.12 & 2.86 & II & 0.92 & 0.05 & 0.02 & 0.02 & 0.69 & 0.10 & 0.17 & 0.05 & 0.69 & 0.03 & 0.24 & 0.04 \\
\hline 11.70 & 3.22 & 2.94 & II & 0.91 & 0.05 & 0.03 & 0.02 & 0.69 & 0.10 & 0.16 & 0.05 & 0.68 & 0.02 & 0.25 & 0.04 \\
\hline 12.65 & 3.34 & 3.09 & II & 0.90 & 0.05 & 0.03 & 0.02 & 0.70 & 0.09 & 0.16 & 0.05 & 0.68 & 0.02 & 0.26 & 0.04 \\
\hline 13.65 & 3.46 & 3.32 & II & 0.89 & 0.05 & 0.04 & 0.02 & 0.70 & 0.09 & 0.16 & 0.05 & 0.68 & 0.02 & 0.27 & 0.03 \\
\hline 14.70 & 3.48 & 3.75 & II & 0.89 & 0.05 & 0.04 & 0.02 & 0.70 & 0.09 & 0.16 & 0.05 & 0.68 & 0.02 & 0.27 & 0.03 \\
\hline 15.65 & 3.52 & 4.20 & II & 0.89 & 0.05 & 0.04 & 0.02 & 0.70 & 0.09 & 0.16 & 0.05 & 0.68 & 0.02 & 0.27 & 0.03 \\
\hline
\end{tabular}




\begin{tabular}{|c|c|c|c|c|c|c|c|c|c|c|c|c|c|c|c|}
\hline 16.60 & 3.61 & 4.61 & II & 0.88 & 0.05 & 0.04 & 0.02 & 0.71 & 0.09 & 0.16 & 0.05 & 0.68 & 0.02 & 0.27 & 0.03 \\
\hline 2.75 & 2.14 & 1.52 & II & 0.96 & 0.02 & 0.01 & 0.01 & 0.73 & 0.08 & 0.15 & 0.04 & 0.71 & 0.03 & 0.19 & 0.07 \\
\hline 4.30 & 2.16 & 1.80 & II & 0.96 & 0.02 & 0.01 & 0.01 & 0.73 & 0.08 & 0.15 & 0.04 & 0.72 & 0.03 & 0.18 & 0.07 \\
\hline 4.85 & 2.18 & 1.82 & II & 0.95 & 0.03 & 0.01 & 0.01 & 0.73 & 0.08 & 0.15 & 0.04 & 0.71 & 0.02 & 0.21 & 0.06 \\
\hline 5.90 & 2.21 & 1.98 & II & 0.95 & 0.03 & 0.01 & 0.01 & 0.72 & 0.08 & 0.16 & 0.04 & 0.71 & 0.02 & 0.21 & 0.06 \\
\hline 6.80 & 2.26 & 2.14 & II & 0.95 & 0.03 & 0.01 & 0.01 & 0.72 & 0.08 & 0.16 & 0.04 & 0.71 & 0.02 & 0.22 & 0.05 \\
\hline 7.45 & 2.36 & 2.12 & II & 0.94 & 0.03 & 0.02 & 0.02 & 0.72 & 0.08 & 0.16 & 0.04 & 0.69 & 0.02 & 0.24 & 0.05 \\
\hline 9.20 & 2.53 & 2.31 & II & 0.93 & 0.04 & 0.02 & 0.02 & 0.71 & 0.08 & 0.17 & 0.04 & 0.68 & 0.02 & 0.26 & 0.04 \\
\hline 11.05 & 2.82 & 2.55 & II & 0.91 & 0.04 & 0.03 & 0.02 & 0.71 & 0.07 & 0.18 & 0.04 & 0.67 & 0.02 & 0.27 & 0.04 \\
\hline \multicolumn{2}{|c|}{ AADp } & $13 \%$ & \multicolumn{9}{|c|}{ AADc } & 0.01 & 0.06 & 0.07 & 0.01 \\
\hline
\end{tabular}

443

444

445

446

447

448

449

450

451

452

453

454

455

456

457

458

459

460

461

462

\subsection{Microscopic measurements tools}

In the previous sections, various indirect methods of determining hydrate composition have been reviewed. All approaches were based on measuring fluid and system properties like pressure, temperature, gas phase composition etc. and then calculating the hydrate composition according to the material balance and hydrate properties. Recently, innovative microscopic instruments helped researchers to measure directly the properties and molecular aspects of clathrate hydrates such as hydrate structure, cage occupancy, hydrate composition and guest molecule position.

\subsubsection{Experimental data and simulation results}

One of the first studies using Raman spectroscopy was performed by Sum et al [72] to explore the properties of clathrate hydrates for pure guest molecules such as $\mathrm{CH}_{4}, \mathrm{CO}_{2}, \mathrm{C}_{3} \mathrm{H}_{8}$ and binary mixtures $\mathrm{CH}_{4}-\mathrm{CO}_{2}, \mathrm{CD}_{4}-\mathrm{C}_{3} \mathrm{H}_{8}, \mathrm{CH}_{4}-\mathrm{N}_{2}, \mathrm{CH}_{4}-\mathrm{THF}_{\mathrm{d} 8}$ and $\mathrm{CH}_{4}-\mathrm{C}_{7} \mathrm{D}_{14}$. As to hydrate composition, they only reported the guest composition in hydrate phase for $\mathrm{CH}_{4}-\mathrm{CO}_{2}$ binary mixture. Their procedure included several steps; preparation of hydrate sample in cell and setting the cell into the sample chamber of the Raman. Then, the pressure was adjusted to a value which was within $5 \%$ of the predicted pressure at a desired temperature. They predicted hydrate equilibrium pressure by CSMHYD [60,72]. Spectra were then collected at the equilibrium conditions for hydrate composition analyses. Table 19 presents their experimental data and reciprocal simulation results by the thermodynamic model.

Table 19. Experimental data from Sum et al. [72] and simulation results

\begin{tabular}{|c|c|c|c|c|c|c|c|}
\hline \multirow{2}{*}{$\mathrm{T}(\mathrm{K})$} & \multicolumn{3}{|c|}{ Gas composition (exp) } & \multirow{2}{*}{$\mathrm{S}$} & \multicolumn{2}{|c|}{$\begin{array}{c}\text { Hydrate composition (exp) } \\
( \pm 1 \%)\end{array}$} & \multicolumn{2}{c|}{ Hydrate composition (pre) } \\
\cline { 2 - 3 } \cline { 6 - 8 } & $\mathrm{CO} 2$ & $\mathrm{CH} 4$ & & $\mathrm{CO} 2$ & $\mathrm{CH} 4$ & $\mathrm{CO} 2$ & $\mathrm{CH} 4$ \\
\hline 273.15 & 0.57 & 0.43 & $\mathrm{I}$ & 0.75 & 0.25 & 0.73 & 0.27 \\
\hline 274.15 & 0.57 & 0.43 & $\mathrm{I}$ & 0.73 & 0.27 & 0.73 & 0.27 \\
\hline 277.15 & 0.57 & 0.43 & $\mathrm{I}$ & 0.71 & 0.29 & 0.72 & 0.28 \\
\hline 278.15 & 0.57 & 0.43 & $\mathrm{I}$ & 0.71 & 0.29 & 0.72 & 0.28 \\
\hline 273.15 & 0.34 & 0.66 & $\mathrm{I}$ & 0.55 & 0.45 & 0.52 & 0.48 \\
\hline
\end{tabular}




\begin{tabular}{|l|l|l|l|l|l|l|l|}
\hline 274.15 & 0.34 & 0.66 & I & 0.55 & 0.45 & 0.51 & 0.49 \\
\hline 275.15 & 0.34 & 0.66 & I & 0.53 & 0.47 & 0.51 & 0.49 \\
\hline 278.15 & 0.34 & 0.66 & I & 0.52 & 0.48 & 0.50 & 0.50 \\
\hline \multicolumn{2}{|r|}{} & AADc & \multicolumn{2}{|c|}{0.02} \\
\hline
\end{tabular}

464 Table 19 illustrates that the thermodynamic model satisfactorily simulated the hydrate 465 composition. The average absolute deviation for hydrate composition was 0.02 .

466 Subramanian et al. studied the structural transition of methane-ethane hydrate mixture based on 467 Raman spectroscopy and ${ }^{13} \mathrm{C}$ NMR measurements [73,74]. According to the Raman spectra, they 468 reported that for methane-ethane hydrate mixture at $274.2 \mathrm{~K}$, there was a structural transition 469 from structure I to II, when the methane composition in gas phase was between 0.722 and 0.750 470 (mole fraction). Moreover, they observed that the structure changed from sII to sI, once the 471 methane composition in vapor phase was between 0.992 and 0.994 (mole fraction). Furthermore, 472 Raman spectra showed that structural transition leads to a $20 \%$ variation in hydrate composition. 473 They also measured the hydrate composition by ${ }^{13} \mathrm{C} N \mathrm{NM}$ at six different vapor compositions. 474 Their results are listed in Table 20.

Table 20. Experimental data from Subramanian et al. [74] for methane-ethane mixture and simulation results

\begin{tabular}{|c|c|c|c|c|c|c|c|c|c|}
\hline \multirow{2}{*}{$\mathrm{T}(\mathrm{K})$} & \multirow{2}{*}{$\begin{array}{c}\text { Pexp } \\
(\mathrm{MPa}) \\
( \pm 0.014)\end{array}$} & \multirow{2}{*}{$\begin{array}{l}\text { Ppre } \\
(\mathrm{MPa})\end{array}$} & \multirow{2}{*}{ S } & \multicolumn{2}{|c|}{ Gas composition (exp) } & \multicolumn{2}{|c|}{$\begin{array}{l}\text { Hydrate composition } \\
\quad(\exp )( \pm 0.1 \%)\end{array}$} & \multicolumn{2}{|c|}{$\begin{array}{l}\text { Hydrate composition } \\
\text { (Pre) }\end{array}$} \\
\hline & & & & $\mathrm{CH}_{4}$ & $\mathrm{C}_{2} \mathrm{H}_{6}$ & $\mathrm{CH}_{4}$ & $\mathrm{C}_{2} \mathrm{H}_{6}$ & $\mathrm{CH}_{4}$ & $\mathrm{C}_{2} \mathrm{H}_{6}$ \\
\hline 274.2 & 0.88 & 0.82 & I & 0.63 & 0.37 & 0.28 & 0.72 & 0.30 & 0.70 \\
\hline 274.2 & 0.96 & 0.88 & I & 0.68 & 0.32 & 0.36 & 0.64 & 0.32 & 0.68 \\
\hline 274.2 & 0.97 & 0.96 & I & 0.72 & 0.28 & 0.37 & 0.63 & 0.35 & 0.65 \\
\hline \multicolumn{2}{|c|}{ AADp } & $5.3 \%$ & I & \multicolumn{4}{|c|}{ AADc } & \multicolumn{2}{|c|}{0.02} \\
\hline 274.2 & 0.99 & 1.02 & II & 0.75 & 0.25 & 0.57 & 0.43 & 0.37 & 0.63 \\
\hline 274.2 & 1.17 & 1.34 & II & 0.85 & 0.15 & 0.65 & 0.35 & 0.47 & 0.53 \\
\hline 274.2 & 1.45 & 1.76 & II & 0.92 & 0.08 & 0.70 & 0.30 & 0.61 & 0.39 \\
\hline \multicolumn{2}{|c|}{ AADp } & $41.6 \%$ & II & \multicolumn{4}{|c|}{ AADc } & \multicolumn{2}{|c|}{0.09} \\
\hline
\end{tabular}

476

477

478

479

480

481

482

483

484

485
Clearly, in Table 20, the thermodynamic simulation concurs with the experimental results for structure I. While for the structure II, the simulations fail. This reveals that the thermodynamic model cannot be proficient to well evaluate the hydrate equilibrium conditions when the structure transition occurs in the system.

Seo and Lee [75] analyzed the structure and gas distribution of nitrogen-carbon dioxide hydrate at different vapor compositions. They revealed from X-ray diffraction patterns that when carbon dioxide composition in gas phase was between 3 and 20 mole percent, structure sI formed. While for $\mathrm{CO}_{2}$ composition under $1 \%$, the hydrate structure seemed to be sII. Additionally, they measured the hydrate composition by NMR spectroscopic analyses. They noticed that the $\mathrm{CO}_{2}$ 
486 molecules were mostly localized in the large sI cages. Also, the hydrate composition was 487 significantly augmented by a small increase of $\mathrm{CO}_{2}$ in the vapor phase. Table 21 and Figure 12 488 present their experimental and our simulation results for hydrate guest distribution. This is clear 489 from the figure that the results of thermodynamic model for hydrate composition prediction 490 corresponds well with the experimental data (average deviation of hydrate phase prediction is 491 0.02).

Table 21. Experimental results from Seo and Lee [75] and our simulation results

\begin{tabular}{|c|c|c|c|c|c|c|c|c|c|}
\hline \multirow{2}{*}{$\mathrm{T}(\mathrm{K})$} & \multirow{2}{*}{$\begin{array}{l}\text { Pexp } \\
\text { (MPa) }\end{array}$} & \multirow{2}{*}{$\begin{array}{l}\text { Ppre } \\
\text { (MPa) }\end{array}$} & \multirow[t]{2}{*}{$\mathrm{S}$} & \multicolumn{2}{|c|}{ Gas composition (exp) } & \multicolumn{2}{|c|}{$\begin{array}{l}\text { Hydrate composition } \\
(\exp )( \pm 0.1 \%)\end{array}$} & \multicolumn{2}{|c|}{$\begin{array}{l}\text { Hydrate composition } \\
\text { (Pre) }\end{array}$} \\
\hline & & & & $\mathrm{CO}_{2}$ & $\mathrm{~N}_{2}$ & $\mathrm{CO}_{2}$ & $\mathrm{~N}_{2}$ & $\mathrm{CO}_{2}$ & $\mathrm{~N}_{2}$ \\
\hline 272.1 & 14.50 & 13.57 & I & 0.01 & 0.99 & 0.09 & 0.91 & 0.07 & 0.93 \\
\hline 272.1 & 13.00 & 12.11 & I & 0.03 & 0.97 & 0.15 & 0.85 & 0.17 & 0.83 \\
\hline 272.1 & 10.50 & 8.79 & $\mathrm{I}$ & 0.10 & 0.90 & 0.47 & 0.54 & 0.41 & 0.59 \\
\hline 272.1 & 7.70 & 6.24 & $\mathrm{I}$ & 0.18 & 0.82 & 0.59 & 0.41 & 0.61 & 0.39 \\
\hline 272.1 & 5.00 & 3.99 & I & 0.33 & 0.67 & 0.74 & 0.26 & 0.79 & 0.21 \\
\hline 272.1 & 4.10 & 2.80 & I & 0.50 & 0.50 & 0.86 & 0.14 & 0.88 & 0.12 \\
\hline 272.1 & 3.50 & 2.14 & $\mathrm{I}$ & 0.67 & 0.34 & 0.94 & 0.06 & 0.94 & 0.06 \\
\hline 272.1 & 3.20 & 1.69 & $\mathrm{I}$ & 0.85 & 0.15 & 0.97 & 0.04 & 0.98 & 0.02 \\
\hline \multicolumn{2}{|c|}{ AADp } & $23.3 \%$ & & \multicolumn{4}{|c|}{$\mathrm{AADc}$} & \multicolumn{2}{|c|}{0.02} \\
\hline
\end{tabular}

493 


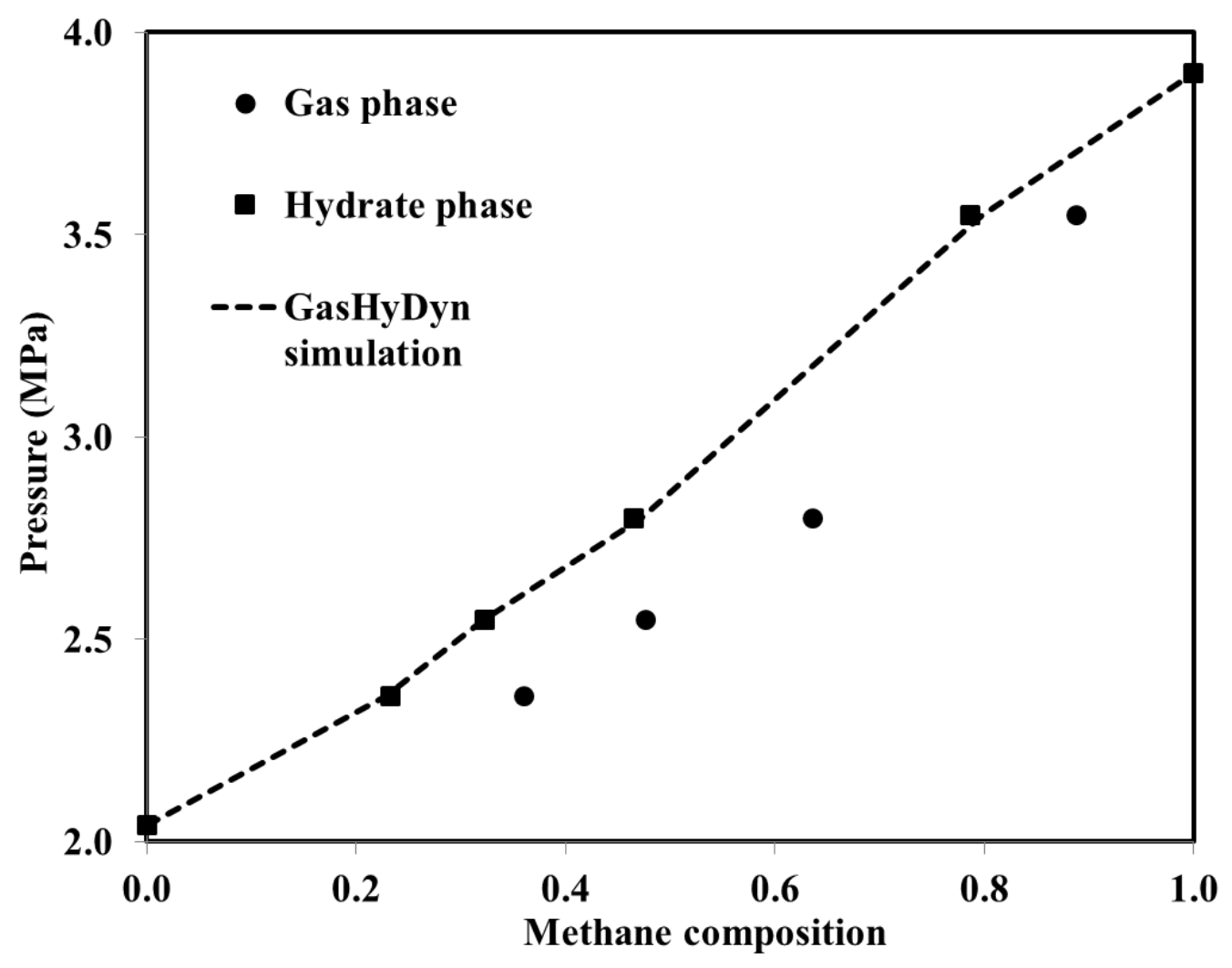

Figure 12. $\mathrm{CO}_{2}$ composition in gas and hydrate phase versus pressure for $\mathrm{CO}_{2}-\mathrm{N}_{2}$ system at $272.1 \mathrm{~K}$ (sI). Experimental [75] and our simulation results

497 Uchida et al. [76] investigated cage occupancy, hydrate composition and structure of methaneethane mixture by Raman spectroscopy and XRD analyses [76]. The prepared the mixed methane-ethane hydrates from ice powder at different feed guest compositions. In the case of hydrate structure by Raman spectroscopy, they reported that, when the composition of ethane in the gas phase was below $2 \%$ or over $22 \%$ mole fraction, only structure I exists. However, there was a coexistence of both structures I and II at ethane concentrations between 12 and $22 \%$ mole fraction. Results of XRD analyses confirmed these investigations. They also observed that ethane molecules were only encapsulated in large cavities, while the methane molecules occupied both small and large cavities. Interestingly, their results revealed that the vapor composition had a significant influence on the cage occupancy in large cavities [76].

Uchida et al. [77] expanded their experiments for $\mathrm{C}_{2} \mathrm{H}_{6}-\mathrm{C}_{3} \mathrm{H}_{8}, \mathrm{CH}_{4}-\mathrm{C}_{2} \mathrm{H}_{6}-\mathrm{C}_{3} \mathrm{H}_{8}$ and $\mathrm{CH}_{4}-\mathrm{C}_{2} \mathrm{H}_{6}$ $\mathrm{C}_{3} \mathrm{H}_{8}-\mathrm{iC}_{4} \mathrm{H}_{10}$ mixed hydrates. Based on the Raman spectra and confirmation of $\mathrm{X}$-ray diffraction, they reported that for $\mathrm{C}_{2} \mathrm{H}_{6}-\mathrm{C}_{3} \mathrm{H}_{8}$ mixed hydrates, only structure II existed when the vapor composition of ethane ranged from 28 to $73 \%$ mole fraction. They also stated that both molecules occupied the $5^{12} 6^{4}$ cages (sII large cages). Moreover, they concluded that the preferential occupancy of $5^{12} 6^{4}$ cages is $\mathrm{C}_{3} \mathrm{H}_{8}>\mathrm{C}_{2} \mathrm{H}_{6}>\mathrm{CH}_{4}$. For $\mathrm{CH}_{4}-\mathrm{C}_{2} \mathrm{H}_{6}-\mathrm{C}_{3} \mathrm{H}_{8}$ hydrates, the initial methane composition in gas phase was between 90 and $98 \%$ mole fraction. Their work showed that, at 
514 final state, the composition of methane in gas phase increased, whereas the ethane and propane 515 composition decreased. In hydrate phase, propane enriched more than other guest molecules. 516 Furthermore, if the cage occupancy of ethane and propane was less than 33\%, the methane 517 molecules can occupy some $5^{12} 6^{4}$ cages. For the quaternary hydrate mixture, they reported the 518 existence of structure II. In addition, all the molecules except methane, occupied the $5^{12} 6^{4}$ cages and the ratio is $\mathrm{C}_{2} \mathrm{H}_{6}-\mathrm{C}_{3} \mathrm{H}_{8}-\mathrm{iC}_{4} \mathrm{H}_{10}=2: 3: 5$. They concluded that at a desired temperature, the larger molecules with lower dissociation pressure, enriched more in hydrate phase [77].

521 Schicks et al. [78] studied the phase behavior of methane-propane and methane-ethane-propane 522 hydrates in a temperature range between 260 and $290 \mathrm{~K}$ and a pressure range from 1 to $6 \mathrm{MPa}$. 523 They investigated the phase transition, hydrate structure and composition by Raman spectroscopy 524 and x-ray diffraction. They observed two different types of crystals after hydrate formation; light 525 and dark. They reported the presence of occluded gas in the structure of light hydrate crystals. 526 However, there was no evidence of the occluded gas in the dark crystals which was supposed to be structure II. Their approach demonstrated that there was a transformation process near the decomposition line. During the transformation, the crystal formation and decomposition was quick. They suggested that this is due to the occluded of free gas or water. Below this, they observed both structure I and II [78].

\subsubsection{Issues of hydrate composition calculation from cage occupancy}

532 Although there are a lot of studies on the pure hydrate formers or kinetic investigation of mixed hydrates by microscopic measurements tools [79-86], there is still little research based on these tools to measure mixed hydrates composition at equilibrium. Furthermore, even in these pertinent studies, they sometimes did not mention clearly their experimental data on hydrate composition (reporting experimental results only on figures, for instance). Therefore, it was impossible to compare their experimental results with the thermodynamic model. In some studies, researchers measured the relative ratios of cage occupancy for mixtures based on Raman spectroscopy. Then, based on the statistical thermodynamics, they calculated absolute cage occupancy. Hydrate 541 information was retrieved from statistical thermodynamics, they are of less interest for this 542 review.

\section{5. Conclusion}

544 While equilibrium conditions of clathrate hydrates, such as temperature, pressure and gas phase 545 composition have been widely studied, the hydrate composition is usually ignored due to the 546 experimental difficulties, like the non-homogenous hydrate phase, water and free gas occlusion, 547 etc. [12]. However, vital data for hydrate composition can be compiled from literature. This 548 information provides comprehensive, representative, and additional knowledge about hydrate 549 composition according to the different equilibrium conditions. Hence, this was the motivation to 550 assemble the relevant research on the hydrate composition by different experimental procedures 
551 and compare these methods via simulation based on the van der Waals and Platteeuw approach 552 and Kihara potential for hydrate equilibrium pressure and composition.

553 Hydrate composition of binary mixtures of carbon dioxide, methane and nitrogen were usually 554 studied since the idea of methane exploration from the hydrate resources by carbon dioxide 555 isolation and gas separation was presented. Experimental data indicated that hydrate composition 556 strongly depends on guest composition in gas phase. A small change in gas composition could 557 lead to a substantial change in hydrate composition. Furthermore, the composition of heavier 558 hydrocarbons (propane and butane) in the hydrate phase was significantly higher than in the gas 559 phase.

560 Our simulations showed that generally, the thermodynamic model predicted adequately the 561 hydrate equilibrium composition. In fact, the thermodynamic model had the best accordance with 562 the experimental data obtained by the microscopic tools like Raman spectroscopy. This suggests 563 that using these direct measurement techniques might help researchers to get closer to reality. 564 Nevertheless, the existence of structural transition led to a significant deviation. Failure to predict 565 structure change could be one of the main challenges for applying effectively the thermodynamic 566 models.

567 Furthermore, at pressures higher than $7 \mathrm{MPa}$ or when $\mathrm{CO}_{2}$ was the minor component in hydrate 568 phase, the deviation of the thermodynamic model from the experimental data was considerable.

569 Unfortunately, there were some cases which the agreement between the results of thermodynamic 570 model and experimental data were not satisfactory. This might be explained by kinetic effects 571 during the crystallization as well as the experimental methodology which could have a significant 572 influence on the experiment. Furthermore, Bouillot and Herri reported that a small change of 573 Kihara parameters had a considerable effect on the hydrate pressure and composition calculations

574 [58]. Hence, it is essential to extend the experimental database in order to well optimize the 575 Kihara parameters. Interestingly, there is still a lack of data concerning storage capacity of 576 hydrates. This information could be used to re-design and develop chemical processes which the 577 volume of gas stored in hydrate phase has been taken into account. Finally, consistent, reliable 578 and extensive experimental data is still needed to examine the ability of thermodynamic modeling 579 to predict hydrate composition as well as its developments.

\section{Acknowledgements}

581 The authors would like to thank Professor Christopher Yukna for his help in proofreading and his 582 suggestions. Saheb Maghsoodloo Babakhani and Son Ho-Van are grateful to Ecole des Mines de 583 Saint-Etienne for providing them Ph.D. scholarships.

\section{References}


[1] W.K. Burton, N. Cabrera, F.C. Frank, The Growth of Crystals and the Equilibrium Structure of their Surfaces, Philos. Trans. R. Soc. Math. Phys. Eng. Sci. 243 (1951) 299-358. doi:10.1098/rsta.1951.0006.

[2] P.B. Dharmawardhana, W.R. Parrish, E.D. Sloan, Experimental Thermodynamic Parameters for the Prediction of Natural Gas Hydrate Dissociation Conditions, Ind. Eng. Chem. Fundam. 19 (1980) 410-414. doi:10.1021/i160076a015.

[3] P. Englezos, N. Kalogerakis, P.D. Dholabhai, P.R. Bishnoi, Kinetics of formation of methane and ethane gas hydrates, Chem. Eng. Sci. 42 (1987) 2647-2658. doi:10.1016/00092509(87)87015-X.

[4] J.-M. Herri, F. Gruy, J.-S. Pic, M. Cournil, B. Cingotti, A. Sinquin, Interest of in situ turbidimetry for the characterization of methane hydrate crystallization: application to the study of kinetic inhibitors, Chem. Eng. Sci. 54 (1999a) 1849-1858.

[5] A.K.M. Jamaluddin, N. Kalogerakis, P.R. Bishnoi, Hydrate plugging problems in undersea natural gas pipelines under shutdown conditions, J. Pet. Sci. Eng. 5 (1991) 323-335. doi:10.1016/0920-4105(91)90051-N.

[6] T. Maekawa, Phase equilibria for hydrate formation from binary mixtures of ethane, propane and noble gases, Fluid Phase Equilibria. 243 (2006) 115-120. doi:10.1016/j.fluid.2006.02.015.

[7] H.-J. Ng, D.B. Robinson, A Method for Predicting the Equilibrium Gas Phase Water Content in Gas-Hydrate Equilibrium, Ind. Eng. Chem. Fundam. 19 (1980) 33-36. doi:10.1021/i160073a006.

[8] J. Nixdorf, L.R. Oellrich, Experimental determination of hydrate equilibrium conditions for pure gases, binary and ternary mixtures and natural gases, Fluid Phase Equilibria. 139 (1997) 325-333. doi:10.1016/S0378-3812(97)00141-6.

[9] T. Collett, J.-J. Bahk, R. Baker, R. Boswell, D. Divins, M. Frye, D. Goldberg, J. Husebø, C. Koh, M. Malone, M. Morell, G. Myers, C. Shipp, M. Torres, Methane Hydrates in NatureCurrent Knowledge and Challenges, J. Chem. Eng. Data. 60 (2015) 319-329. doi: $10.1021 / \mathrm{je} 500604 \mathrm{~h}$.

[10] A. Demirbas, Methane hydrates as potential energy resource: Part 2 - Methane production processes from gas hydrates, Energy Convers. Manag. 51 (2010) 1562-1571. doi:10.1016/j.enconman.2010.02.014.

[11] Y.F. Makogon, Natural gas hydrates - A promising source of energy, J. Nat. Gas Sci. Eng. 2 (2010) 49-59. doi:10.1016/j.jngse.2009.12.004.

[12] E.D. Sloan, C.A. Koh, Clathrate Hydrates of Natural Gases, 3rd ed., CRC Press, 2007.

[13] N.H. Duc, F. Chauvy, J.-M. Herri, CO2 capture by hydrate crystallization - A potential solution for gas emission of steelmaking industry, Energy Convers. Manag. 48 (2007) 1313-1322. doi:10.1016/j.enconman.2006.09.024.

[14] J.-M. Herri, A. Bouchemoua, M. Kwaterski, A. Fezoua, Y. Ouabbas, A. Cameirao, Gas hydrate equilibria for $\mathrm{CO} 2-\mathrm{N} 2$ and $\mathrm{CO} 2-\mathrm{CH} 4$ gas mixtures-Experimental studies and thermodynamic modelling, Fluid Phase Equilibria. 301 (2011) 171-190. doi:10.1016/j.fluid.2010.09.041.

[15] R. Kumar, H. Wu, P. Englezos, Incipient hydrate phase equilibrium for gas mixtures containing hydrogen, carbon dioxide and propane, Fluid Phase Equilibria. 244 (2006) 167171. doi:10.1016/j.fluid.2006.04.008.

[16] P. Linga, R. Kumar, P. Englezos, The clathrate hydrate process for post and pre-combustion capture of carbon dioxide, J. Hazard. Mater. 149 (2007) 625-629. doi:10.1016/j.jhazmat.2007.06.086. 
632

633

634

635

636

637

638

639

640

641

642

643

644

645

646

647

648

649

650

651

652

653

654

655

656

657

658

659

660

661

662

663

664

665

666

667

668

669

670

671

672

673

674

675

676

677

[17] J. Park, Y.-T. Seo, J. Lee, H. Lee, Spectroscopic analysis of carbon dioxide and nitrogen mixed gas hydrates in silica gel for CO2 separation, Catal. Today. 115 (2006) 279-282. doi:10.1016/j.cattod.2006.02.059.

[18] C.-Y. Sun, G.-J. Chen, L.-W. Zhang, Hydrate phase equilibrium and structure for (methane + ethane + tetrahydrofuran + water) system, J. Chem. Thermodyn. 42 (2010) 1173-1179. doi:10.1016/j.jct.2010.04.021.

[19] Q. Sun, X. Guo, A. Liu, B. Liu, Y. Huo, G. Chen, Experimental Study on the Separation of CH4 and N2 via Hydrate Formation in TBAB Solution, Ind. Eng. Chem. Res. 50 (2011) 2284-2288. doi:10.1021/ie101726f.

[20] H. Tajima, A. Yamasaki, F. Kiyono, Energy consumption estimation for greenhouse gas separation processes by clathrate hydrate formation, Energy. 29 (2004) 1713-1729. doi:10.1016/j.energy.2004.03.003.

[21] V.M. Vorotyntsev, V.M. Malyshev, P.G. Taraburov, G.M. Mochalov, Separation of Gas Mixtures by Continuous Gas Hydrate Crystallization, Theor. Found. Chem. Eng. 35 (2001) 513-515. doi:10.1023/A:1012390423740.

[22] S.-P. Kang, J. Lee, Y. Seo, Pre-combustion capture of CO2 by gas hydrate formation in silica gel pore structure, Chem. Eng. J. 218 (2013) 126-132. doi:10.1016/j.cej.2012.11.131.

[23] Q.-L. Ma, G.-J. Chen, C.-Y. Sun, Vapor-liquid-liquid-hydrate phase equilibrium calculation for multicomponent systems containing hydrogen, Fluid Phase Equilibria. 338 (2013) 87-94. doi:10.1016/j.fluid.2012.11.002.

[24] S. Said, V. Govindaraj, J.-M. Herri, Y. Ouabbas, M. Khodja, M. Belloum, J.S. Sangwai, R. Nagarajan, A study on the influence of nanofluids on gas hydrate formation kinetics and their potential: Application to the CO2 capture process, J. Nat. Gas Sci. Eng. 32 (2016) 95108. doi:10.1016/j.jngse.2016.04.003.

[25] M. Yang, W. Jing, J. Zhao, Z. Ling, Y. Song, Promotion of hydrate-based CO2 capture from flue gas by additive mixtures (THF (tetrahydrofuran) + TBAB (tetra-n-butyl ammonium bromide)), Energy. 106 (2016) 546-553. doi:10.1016/j.energy.2016.03.092.

[26] M. Yang, Y. Song, L. Jiang, Y. Zhao, X. Ruan, Y. Zhang, S. Wang, Hydrate-based technology for CO2 capture from fossil fuel power plants, Appl. Energy. 116 (2014) 26-40. doi:10.1016/j.apenergy.2013.11.031.

[27] T. Yu, T. Sato, T. Nakashima, M. Inui, H. Oyama, An integrated model for CO2 hydrate formation in sand sediments for sub-seabed $\mathrm{CO} 2$ storage, Int. J. Greenh. Gas Control. 52 (2016) 250-269. doi:10.1016/j.ijggc.2016.07.009.

[28] J. Zheng, P. Zhang, P. Linga, Semiclathrate hydrate process for pre-combustion capture of CO2 at near ambient temperatures, Appl. Energy. 194 (2017) 267-278. doi:10.1016/j.apenergy.2016.10.118.

[29] J. Zheng, Y.K. Lee, P. Babu, P. Zhang, P. Linga, Impact of fixed bed reactor orientation, liquid saturation, bed volume and temperature on the clathrate hydrate process for precombustion carbon capture, J. Nat. Gas Sci. Eng. 35, Part B (2016) 1499-1510. doi:10.1016/j.jngse.2016.03.100.

[30] D.-L. Zhong, J.-L. Wang, Y.-Y. Lu, Z. Li, J. Yan, Precombustion CO2 capture using a hybrid process of adsorption and gas hydrate formation, Energy. 102 (2016) 621-629. doi:10.1016/j.energy.2016.02.135.

[31] P. Babu, R. Kumar, P. Linga, Unusual behavior of propane as a co-guest during hydrate formation in silica sand: Potential application to seawater desalination and carbon dioxide capture, Chem. Eng. Sci. 117 (2014) 342-351. doi:10.1016/j.ces.2014.06.044. 
678

679

680

681

682

683

684

685

686

687

688

689

690

691

692

693

694

695

696

697

698

699

700

701

702

703

704

705

706

707

708

709

710

711

712

713

714

715

716

717

718

719

720

721

722

723

724

[32] H. Fakharian, H. Ganji, A. Naderifar, Desalination of high salinity produced water using natural gas hydrate, J. Taiwan Inst. Chem. Eng. 72 (2017) 157-162. doi:10.1016/j.jtice.2017.01.025.

[33] S. Han, Y.-W. Rhee, S.-P. Kang, Investigation of salt removal using cyclopentane hydrate formation and washing treatment for seawater desalination, Desalination. 404 (2017) 132137. doi:10.1016/j.desal.2016.11.016.

[34] K.C. Kang, P. Linga, K. Park, S.-J. Choi, J.D. Lee, Seawater desalination by gas hydrate process and removal characteristics of dissolved ions $\left(\mathrm{Na}^{+}, \mathrm{K}+, \mathrm{Mg} 2+, \mathrm{Ca} 2+, \mathrm{B} 3+, \mathrm{Cl}^{-}\right.$, SO42 -), Desalination. 353 (2014) 84-90. doi:10.1016/j.desal.2014.09.007.

[35] Y.-N. Lv, S.-S. Wang, C.-Y. Sun, J. Gong, G.-J. Chen, Desalination by forming hydrate from brine in cyclopentane dispersion system, Desalination. 413 (2017) 217-222. doi:10.1016/j.desal.2017.03.025.

[36] M. Yang, J. Zheng, W. Liu, Y. Liu, Y. Song, Effects of C3H8 on hydrate formation and dissociation for integrated CO2 capture and desalination technology, Energy. 93, Part 2 (2015) 1971-1979. doi:10.1016/j.energy.2015.10.076.

[37] J. Zheng, M. Yang, Y. Liu, D. Wang, Y. Song, Effects of cyclopentane on CO2 hydrate formation and dissociation as a co-guest molecule for desalination, J. Chem. Thermodyn. 104 (2017) 9-15. doi:10.1016/j.jct.2016.09.006.

[38] S. Ho-Van, B. Bouillot, J. Douzet, S.M. Babakhani, J.M. Herri, Experimental measurement and thermodynamic modeling of cyclopentane hydrates with $\mathrm{NaCl}, \mathrm{KCl}, \mathrm{CaCl} 2$, or NaCl$\mathrm{KCl}$ present, AIChE J. In press (2018). doi:10.1002/aic.16067.

[39] A. Erfani, E. Fallah-Jokandan, F. Varaminian, Effects of non-ionic surfactants on formation kinetics of structure $\mathrm{H}$ hydrate regarding transportation and storage of natural gas, J. Nat. Gas Sci. Eng. 37 (2017) 397-408. doi:10.1016/j.jngse.2016.11.058.

[40] J. Javanmardi, K. Nasrifar, S.H. Najibi, M. Moshfeghian, Economic evaluation of natural gas hydrate as an alternative for natural gas transportation, Appl. Therm. Eng. 25 (2005) 1708-1723. doi:10.1016/j.applthermaleng.2004.10.009.

[41] J. Lim, E. Kim, Y. Seo, Dual inhibition effects of diamines on the formation of methane gas hydrate and their significance for natural gas production and transportation, Energy Convers. Manag. 124 (2016) 578-586. doi:10.1016/j.enconman.2016.07.054.

[42] S. Maghsoodloo Babakhani, A. Alamdari, Effect of maize starch on methane hydrate formation/dissociation rates and stability, J. Nat. Gas Sci. Eng. 26 (2015) 1-5. doi:10.1016/j.jngse.2015.05.026.

[43] H. Mimachi, S. Takeya, A. Yoneyama, K. Hyodo, T. Takeda, Y. Gotoh, T. Murayama, Natural gas storage and transportation within gas hydrate of smaller particle: Size dependence of self-preservation phenomenon of natural gas hydrate, Chem. Eng. Sci. 118 (2014) 208-213. doi:10.1016/j.ces.2014.07.050.

[44] Z. Taheri, M.R. Shabani, K. Nazari, A. Mehdizaheh, Natural gas transportation and storage by hydrate technology: Iran case study, J. Nat. Gas Sci. Eng. 21 (2014) 846-849. doi:10.1016/j.jngse.2014.09.026.

[45] I. Chatti, A. Delahaye, L. Fournaison, J.-P. Petitet, Benefits and drawbacks of clathrate hydrates: a review of their areas of interest, Energy Convers. Manag. 46 (2005) 1333-1343. doi:10.1016/j.enconman.2004.06.032.

[46] J.-M. Herri, M. Cournil, E. Chassefiere, Thermodynamic modelling of clathrate hydrates in the atmosphere of Mars, HAL. (2011). https://hal.archives-ouvertes.fr/hal-00617450.

[47] J.-M. Herri, E. Chassefière, Carbon dioxide, argon, nitrogen and methane clathrate hydrates: Thermodynamic modelling, investigation of their stability in Martian atmospheric 
conditions and variability of methane trapping, Planet. Space Sci. 73 (2012) 376-386. doi:10.1016/j.pss.2012.07.028.

[48] S.L. Miller, W.D. Smythe, Carbon dioxide clathrate in the martian ice cap, Science. 170 (1970) 531-533. doi:10.1126/science.170.3957.531.

[49] M.N. Khan, P. Warrier, C.J. Peters, C.A. Koh, Review of vapor-liquid equilibria of gas hydrate formers and phase equilibria of hydrates, J. Nat. Gas Sci. Eng. 35, Part B (2016) 1388-1404. doi:10.1016/j.jngse.2016.06.043.

[50] A.Y. Manakov, A.D. Duchkov, Laboratory modeling of hydrate formation in rock specimens (a review), Russ. Geol. Geophys. 58 (2017) 240-252. doi:10.1016/j.rgg.2016.01.023.

[51] S. Shahnazar, N. Hasan, Gas hydrate formation condition: Review on experimental and modeling approaches, Fluid Phase Equilibria. 379 (2014) 72-85. doi:10.1016/j.fluid.2014.07.012.

[52] Y. Song, L. Yang, J. Zhao, W. Liu, M. Yang, Y. Li, Y. Liu, Q. Li, The status of natural gas hydrate research in China: A review, Renew. Sustain. Energy Rev. 31 (2014) 778-791. doi:10.1016/j.rser.2013.12.025.

[53] Q. Sun, Y.T. Kang, Review on $\mathrm{CO} 2$ hydrate formation/dissociation and its cold energy application, Renew. Sustain. Energy Rev. 62 (2016) 478-494. doi:10.1016/j.rser.2016.04.062.

[54] Z. Yin, Z.R. Chong, H.K. Tan, P. Linga, Review of gas hydrate dissociation kinetic models for energy recovery, J. Nat. Gas Sci. Eng. 35, Part B (2016) 1362-1387. doi:10.1016/j.jngse.2016.04.050.

[55] J.H. van der Waals, J.C. Platteeuw, Clathrate Solutions, in: I. Prigogine (Ed.), Adv. Chem. Phys., John Wiley \& Sons, Inc., Hoboken, NJ, USA, 1959: pp. 1-57. http://doi.wiley.com/10.1002/9780470143483.ch1 (accessed December 22, 2015).

[56] Y.P. Handa, J.S. Tse, Thermodynamic properties of empty lattices of structure I and structure II clathrate hydrates, J. Phys. Chem. 90 (1986) 5917-5921. doi:10.1021/j100280a092.

[57] D. Le Quang, D. Le Quang, B. Bouillot, J.-M. Herri, P. Glenat, P. Duchet-Suchaux, Experimental procedure and results to measure the composition of gas hydrate, during crystallization and at equilibrium, from $\mathrm{N} 2-\mathrm{CO} 2-\mathrm{CH} 4-\mathrm{C} 2 \mathrm{H} 6-\mathrm{C} 3 \mathrm{H} 8-\mathrm{C} 4 \mathrm{H} 10$ gas mixtures, Fluid Phase Equilibria. (2015). doi:10.1016/j.fluid.2015.10.022.

[58] B. Bouillot, J.-M. Herri, Framework for clathrate hydrate flash calculations and implications on the crystal structure and final equilibrium of mixed hydrates, Fluid Phase Equilibria. (2015). doi:10.1016/j.fluid.2015.10.023.

[59] V. McKoy, O. Sinanoğlu, Theory of Dissociation Pressures of Some Gas Hydrates, J. Chem. Phys. 38 (1963) 2946. doi:10.1063/1.1733625.

[60] J.E.D. Sloan, Clathrate Hydrates of Natural Gases, Second Edition, Revised and Expanded, CRC Press, 1998.

[61] S. Maghsoodloo Babakhani, B. Bouillot, J. Douzet, S. Ho-Van, J.M. Herri, A new approach of studying mixed gas hydrates involving propane at non-equilibrium conditions and final state: An experimental study and modeling, Chem. Eng. Sci. (2018). doi:10.1016/j.ces.2018.01.017.

[62] J. Jhaveri, D.B. Robinson, Hydrates in the methane-nitrogen system, Can. J. Chem. Eng. 43 (1965) 75-78. doi:10.1002/cjce.5450430207. 
[63] T. Kawasaki, K. Kikuchi, D. Terasaki, T. Okui, K. Myata, H. Hirayama, M. Ihara, Composition of Guests in Hydrates from Gas Mixture, in: Yokohama, Japan, 2002: pp. 424427.

[64] S.-P. Kang, H. Lee, C.-S. Lee, W.-M. Sung, Hydrate phase equilibria of the guest mixtures containing CO2, N2 and tetrahydrofuran, Fluid Phase Equilibria. 185 (2001) 101-109. doi:10.1016/S0378-3812(01)00460-5.

[65] K. Ohgaki, K. Takano, H. Sangawa, T. Matsubara, S. Nakano, Methane exploitation by carbon dioxide from gas hydrates. Phase equilibria for $\mathrm{CO} 2-\mathrm{CH} 4$ mixed hydrate system., J. Chem. Eng. Jpn. 29 (1996) 478-483. doi:10.1252/jcej.29.478.

[66] S. Angus, J.B. Armstrong, K.M. de Reuck, R.D. McCarthy, R.T. Jacobsen, W. Wagner, International thermodynamic tables of the fluid state. Methane 5 5, 1978.

[67] S. Angus, B. Armstrong, K.M. de Reuck, International thermodynamic tables of the fluid state: carbon dioxide, Pergamon Press, Oxford; New York, 1976.

[68] V. Belandria, A. Eslamimanesh, A.H. Mohammadi, P. Théveneau, H. Legendre, D. Richon, Compositional Analysis and Hydrate Dissociation Conditions Measurements for Carbon Dioxide + Methane + Water System, Ind. Eng. Chem. Res. 50 (2011) 5783-5794. doi:10.1021/ie101959t.

[69] V. Belandria, A. Eslamimanesh, A.H. Mohammadi, D. Richon, Gas Hydrate Formation in Carbon Dioxide + Nitrogen + Water System: Compositional Analysis of Equilibrium Phases, Ind. Eng. Chem. Res. 50 (2011) 4722-4730. doi:10.1021/ie101635k.

[70] Y.-T. Seo, S.-P. Kang, H. Lee, C.-S. Lee, W.-M. Sung, Hydrate phase equilibria for gas mixtures containing carbon dioxide: A proof-of-concept to carbon dioxide recovery from multicomponent gas stream, Korean J. Chem. Eng. 17 (2000) 659-667.

[71] H.-J. Ng, Hydrate Phase Composition for Multicomponent Gas Mixtures, Ann. N. Y. Acad. Sci. 912 (2000) 1034-1039. doi:10.1111/j.1749-6632.2000.tb06858.x.

[72] A.K. Sum, R.C. Burruss, E.D. Sloan, Measurement of Clathrate Hydrates via Raman Spectroscopy, J. Phys. Chem. B. 101 (1997) 7371-7377. doi:10.1021/jp970768e.

[73] S. Subramanian, A.. Ballard, R.. Kini, S.. Dec, E.. Sloan, Structural transitions in methane+ethane gas hydrates - Part I: upper transition point and applications, Chem. Eng. Sci. 55 (2000) 5763-5771. doi:10.1016/S0009-2509(00)00162-7.

[74] S. Subramanian, R.A. Kini, S.F. Dec, E.D. Sloan, Evidence of structure II hydrate formation from methane+ethane mixtures, Chem. Eng. Sci. 55 (2000) 1981-1999. doi:10.1016/S00092509(99)00389-9.

[75] Y.-T. Seo, H. Lee, Structure and Guest Distribution of the Mixed Carbon Dioxide and Nitrogen Hydrates As Revealed by X-ray Diffraction and 13C NMR Spectroscopy, J. Phys. Chem. B. 108 (2004) 530-534. doi:10.1021/jp0351371.

[76] T. Uchida, S. Takeya, Y. Kamata, I.Y. Ikeda, J. Nagao, T. Ebinuma, H. Narita, O. Zatsepina, B.A. Buffett, Spectroscopic Observations and Thermodynamic Calculations on Clathrate Hydrates of Mixed Gas Containing Methane and Ethane: Determination of Structure, Composition and Cage Occupancy, J. Phys. Chem. B. 106 (2002) 12426-12431. doi:10.1021/jp025884i.

[77] T. Uchida, S. Takeya, Y. Kamata, R. Ohmura, H. Narita, Spectroscopic Measurements on Binary, Ternary, and Quaternary Mixed-Gas Molecules in Clathrate Structures, Ind. Eng. Chem. Res. 46 (2007) 5080-5087. doi:10.1021/ie070153w.

[78] J.M. Schicks, R. Naumann, J. Erzinger, K.C. Hester, C.A. Koh, E.D. Sloan, Phase Transitions in Mixed Gas Hydrates: Experimental Observations versus Calculated Data, J. Phys. Chem. B. 110 (2006) 11468-11474. doi:10.1021/jp0612580. 
[79] D.W. Davidson, S.K. Garg, S.R. Gough, R.E. Hawkins, J.A. Ripmeester, Characterization of natural gas hydrates by nuclear magnetic resonance and dielectric relaxation, Can. J. Chem. 55 (1977) 3641-3650. doi:10.1139/v77-512.

[80] J.A. Ripmeester, C.I. Ratcliffe, The Diverse Nature of Dodecahedral Cages in Clathrate Hydrates As Revealed by 129 Xe and 13 C NMR Spectroscopy: CO2 as a Small-Cage Guest, Energy Fuels. 12 (1998) 197-200. doi:10.1021/ef970171y.

[81] S. Nakano, M. Moritoki, K. Ohgaki, High-Pressure Phase Equilibrium and Raman Microprobe Spectroscopic Studies on the CO2 Hydrate System, J. Chem. Eng. Data. 43 (1998) 807-810. doi:10.1021/je9800555.

[82] I.L. Moudrakovski, G.E. McLaurin, C.I. Ratcliffe, J.A. Ripmeester, Methane and Carbon Dioxide Hydrate Formation in Water Droplets: Spatially Resolved Measurements from Magnetic Resonance Microimaging, J. Phys. Chem. B. 108 (2004) 17591-17595. doi: $10.1021 / \mathrm{jp} 0473220$.

[83] S. Subramanian, E.D. Sloan, Microscopic Measurements and Modeling of Hydrate Formation Kinetics, Ann. N. Y. Acad. Sci. 912 (2006) 583-592. doi:10.1111/j.17496632.2000.tb06813.x.

[84] S. Gao, W.G. Chapman, W. House, NMR and Viscosity Investigation of Clathrate Hydrate Formation and Dissociation, Ind. Eng. Chem. Res. 44 (2005) 7373-7379. doi:10.1021/ie050464b.

[85] K.C. Hester, T.A. Strobel, E.D. Sloan, C.A. Koh, A. Huq, A.J. Schultz, Molecular Hydrogen Occupancy in Binary THF-H2 Clathrate Hydrates by High Resolution Neutron Diffraction, J. Phys. Chem. B. 110 (2006) 14024-14027. doi:10.1021/jp063164w.

[86] R. Kumar, P. Englezos, I. Moudrakovski, J.A. Ripmeester, Structure and composition of $\mathrm{CO} 2 / \mathrm{H} 2$ and $\mathrm{CO} 2 / \mathrm{H} 2 / \mathrm{C} 3 \mathrm{H} 8$ hydrate in relation to simultaneous $\mathrm{CO} 2$ capture and $\mathrm{H} 2$ production, AIChE J. 55 (2009) 1584-1594. doi:10.1002/aic.11844. 